\title{
THE PECULIAR CHEMICAL INVENTORY OF NGC 2419: AN EXTREME OUTER HALO “GLOBULAR CLUSTER”*
}

\author{
Judith G. Cohen ${ }^{1}$, Wenjin Huang ${ }^{2}$, And Evan N. Kirby ${ }^{1,3}$ \\ ${ }^{1}$ Palomar Observatory, Mail Stop 249-17, California Institute of Technology, Pasadena, CA 91125, USA; jlc@ astro.caltech.edu; enk@astro.caltech.edu \\ 2 Brion Technologies Inc., 4211 Burton Drive, Santa Clara, CA 95054, USA; wenjin.huang@ @rion.com \\ Received 2011 March 4; accepted 2011 July 12; published 2011 September 29
}

\begin{abstract}
NGC 2419 is a massive outer halo Galactic globular cluster (GC) whose stars have previously been shown to have somewhat peculiar abundance patterns. We have observed seven luminous giants that are members of NGC 2419 with Keck/HIRES at reasonable signal-to-noise ratio. One of these giants is very peculiar, with an extremely low $[\mathrm{Mg} / \mathrm{Fe}]$ and high $[\mathrm{K} / \mathrm{Fe}]$ but normal abundances of most other elements. The abundance pattern does not match the nucleosynthetic yields of any supernova model. The other six stars show abundance ratios typical of inner halo Galactic GCs, represented here by a sample of giants in the nearby GC M30. Although our measurements show that NGC 2419 is unusual in some respects, its bulk properties do not provide compelling evidence for a difference between inner and outer halo GCs.
\end{abstract}

Key words: Galaxy: formation - Galaxy: halo - globular clusters: individual (NGC 2419)

Online-only material: machine-readable tables

\section{INTRODUCTION}

NGC 2419 is a globular cluster (GC) in the outer halo of the Galaxy at a distance of $84 \mathrm{kpc}^{4}$ Baumgardt et al. (2009) determined a velocity dispersion for this cluster based on 40 stars which leads to $M / L=2.05 \pm 0.50 M_{\odot} / L_{\odot}$, a normal value for an old stellar system, with no evidence for dark matter at the present time. Conroy et al. (2010) further asserted that this cluster could not have formed in a now-defunct dark matter halo. Harris et al. (1997) obtained a deep color-magnitude diagram (CMD) of NGC 2419 with Hubble Space Telescope (HST)/ WFPC2 and demonstrated that there is no detectable difference in age between it and M92, an ancient, well-studied inner halo GC of comparable metallicity. Furthermore, there is no evidence from CMDs of multiple stellar populations in NGC 2419.

However, some of the characteristics of NGC 2419 are anomalous for a GC. It has the highest luminosity $\left(M_{V} \sim\right.$ $-9.6 \mathrm{mag}$ ) of any GC with Galactocentric radius $R>20 \mathrm{kpc}$, higher than all other GCs with $R>15 \mathrm{kpc}$ with the exception of M54, which is believed to represent the nuclear region of the Sgr dSph galaxy now being accreted by the Milky Way (Ibata et al. 1995; Sarajedini \& Layden 1995). NGC 2419 also has an unusually large half-light radius $\left(r_{h}=19 \mathrm{pc}\right)$ and core radius $\left(r_{c}=7 \mathrm{pc}\right)$ for a massive Galactic $\mathrm{GC}$ and a relaxation time which exceeds the Hubble time, also unusual for a GC. Every other luminous GC in the outer halo is considerably more compact. The 2010 version of the online database of Harris (1996) lists only three Galactic GCs that lie anomalously above the bulk of the Galactic GCs in the plane $r_{h}$ versus $M_{V}$, one of which is NGC 2419. The other two are M54 and $\omega$ Cen, the most luminous Galactic GC, and one with a large internal

\footnotetext{
* Based in part on observations obtained at the W. M. Keck Observatory, which is operated jointly by the California Institute of Technology, the University of California, and the National Aeronautics and Space Administration.

3 Hubble Fellow.

4 The distance and other parameters for NGC 2419 are from the 2010 version of the online GC database of Harris (1996), based on the photometry of Harris et al. (1997).
}

spread in metallicity, also believed to be the stripped core of a dwarf galaxy accreted by the Milky Way. For these reasons, van den Bergh \& Mackey (2004) and Mackey \& van den Bergh (2005), among others, suggested that NGC 2419 is also the remnant of an accreted dwarf galaxy. In fact, Irwin (1999) and Newberg et al. (2003) suggested that NGC 2419 is one of many GCs associated with the disrupted Sgr dSph, but Law \& Majewski (2010) firmly concluded that it is not. Therefore, the cluster is potentially a piece of an unidentified, tidally disrupted dSph.

To follow up on these earlier suggestions, Cohen et al. (2010) obtained moderate resolution spectra of a large sample of luminous giants in NGC 2419 and analyzed line strengths in the region of the near-infrared $\mathrm{Ca}$ triplet. We found that there is a small but measurable spread in Ca abundance of $\sim 0.3 \mathrm{dex}$ within this massive metal-poor GC. We present here an analysis of high dispersion spectra of seven luminous giant members of NGC 2419, whose chemical inventory we compare to that inferred from a similar sized sample of luminous red giant branch (RGB) members of the nearby inner halo globular cluster M30 (NGC 7099), observed and analyzed in the same way as the NGC 2419 stars. Note that these two GCs have the same metallicity in the 2003 version of the online database of Harris (1996). We explore the following two issues.

1. Is the chemical inventory in such a distant cluster identical to that of a GC of similar $[\mathrm{Fe} / \mathrm{H}]$ in the inner halo?

2. What star-to-star variations are revealed by our highdispersion spectra?

\section{OBSERVATIONS AND ANALYSIS}

We obtained high-spectral-resolution and reasonably high signal-to-noise ratio $(\mathrm{S} / \mathrm{N})$ spectra of seven luminous giants in NGC 2419 using HIRESr (Vogt et al. 1994) on the Keck I $10 \mathrm{~m}$ Telescope. The stars were selected from both the early spectroscopic study of Suntzeff et al. (1988) and the current version of the online photometric database described by Stetson (2005). The sample stars range in $V$ from 17.2 to 17.6 mag. The spectral range is $3900-8350 \AA$ with gaps between the reddest 
Table 1

Data for Red Giant Members of NGC 2419 and of NGC 7099 with HIRES/Keck Spectra

\begin{tabular}{|c|c|c|c|c|c|c|c|}
\hline Name $^{\mathrm{a}}$ & $\begin{array}{c}V^{\mathrm{a}} \\
(\mathrm{mag})\end{array}$ & $\begin{array}{c}T_{\text {eff }}, \log (g), v_{t}^{\mathrm{b}} \\
\left(\mathrm{K}, \operatorname{dex}, \mathrm{km} \mathrm{s}^{-1}\right)\end{array}$ & $\begin{array}{c}{[\mathrm{Fe} / \mathrm{H}](\mathrm{Fe} \mathrm{I})} \\
(\mathrm{dex})\end{array}$ & Date $^{c}$ & $\begin{array}{l}\text { Exp. Time } \\
\text { (s) }\end{array}$ & $\mathrm{S} / \mathrm{N}^{\mathrm{d}}$ & $\begin{array}{c}v_{r} \\
\left(\mathrm{~km} \mathrm{~s}^{-1}\right)\end{array}$ \\
\hline \multicolumn{8}{|l|}{ NGC 2419} \\
\hline Stet $223^{\mathrm{e}}$ & 17.25 & $4250,0.50,2.4$ & -2.09 & $2 / 2010$ & 8400 & $>100$ & -22.4 \\
\hline Stet 810 & 17.31 & $4330,0.60,2.1$ & -2.00 & $11 / 2010$ & 7200 & $>100$ & -22.6 \\
\hline Stet $973^{\mathrm{f}}$ & 17.45 & $4320,0.70,2.1$ & -1.98 & 9/2006 & 3200 & 39 & -21.9 \\
\hline Stet 1131 & 17.61 & $4435,0.75,2.2$ & -1.95 & $2 / 2010$ & 9000 & 95 & -16.8 \\
\hline Stet $1209^{\mathrm{g}}$ & 17.41 & $4350,0.40,2.1$ & -2.20 & $11 / 2010$ & 7200 & 93 & -19.0 \\
\hline Stet $1305^{\mathrm{h}}$ & 17.61 & $4400,0.80,2.3$ & -2.17 & 9/2008 & 3000 & 52 & -16.8 \\
\hline Stet $1814^{\mathrm{i}}$ & 17.27 & $4370,0.55,2.3$ & -2.04 & $2 / 2010$ & 5400 & 90 & -26.1 \\
\hline \multicolumn{8}{|l|}{ NGC 7099} \\
\hline Stet 31 & 13.92 & $4790,1.63,2.2$ & -2.39 & $9 / 2006$ & 1200 & $>100$ & -185.8 \\
\hline Stet 34 & 13.57 & $4658,1.13,2.0$ & -2.32 & $9 / 2006$ & 1200 & $>100$ & -188.1 \\
\hline AL $38^{\mathrm{j}}$ & $13.27^{\mathrm{k}}$ & $4653,1.10,2.1$ & -2.47 & $9 / 2006$ & 900 & $>100$ & -185.6 \\
\hline Stet 42 & 14.70 & $4935,2.03,1.8$ & -2.46 & $9 / 2006$ & 1200 & $>100$ & -190.1 \\
\hline Stet $81^{1}$ & 14.25 & $5050,1.70,2.2$ & -2.48 & $9 / 2006$ & 1200 & $>100$ & -185.9 \\
\hline
\end{tabular}

Notes.

a Star IDs and $V$ mags are from the online version of the database of Stetson (2005).

$\mathrm{b}$ These are the values adopted for the abundance analysis.

${ }^{c}$ Date of the best spectrum. Earlier low S/N spectra were taken for several of the NGC 2419 stars.

${ }^{\mathrm{d}} \mathrm{S} / \mathrm{N}$ per spectral resolution element in the continuum at $5500 \AA$ A.

${ }^{\mathrm{e}}$ Stetson $223=$ Suntzeff 1 .

${ }^{\mathrm{f}}$ Stetson $973=$ Suntzeff 15 .

${ }^{\mathrm{g}}$ Stetson $1209=$ Suntzeff 16.

${ }^{\mathrm{h}}$ Stetson $1305=$ RH 10 (M. Shetrone 2009, private communication), previously observed by Shetrone et al. (2001).

${ }^{\mathrm{i}}$ Stetson $1814=$ Suntzeff 14.

${ }^{j}$ ID from Alcaino \& Liller (1980).

${ }^{\mathrm{k}} V$ mag from Bolte (1987).

${ }^{1}$ S81 lies slightly bluer in $B-V$ than the mean RGB locus.

echelle orders. We used a 1.1 arcsec slit, equivalent to 6.3 pixels at $15 \mu \mathrm{pixel}^{-1}$, to achieve a spectral resolution of 34,000 . The maximum total exposure time for one star was $2.5 \mathrm{hr}$, split into shorter segments to improve cosmic ray removal. The code MAKEE $^{5}$ was used to reduce the HIRES spectra, which were obtained in various runs over the past five years. The majority of the seven spectra have an $\mathrm{S} / \mathrm{N}$ exceeding 90 per spectral resolution element at $5500 \AA$ in the continuum. The $\mathrm{S} / \mathrm{N}$ degrades toward the blue, and we often eliminated lines bluer than $5000 \AA$ for species with many detected features. The radial velocities for these seven stars have a mean of $-20.6 \mathrm{~km} \mathrm{~s}^{-1}$ with $\sigma$ of $3.7 \mathrm{~km} \mathrm{~s}^{-1}$. Cohen et al. (2010) discussed membership issues for NGC 2419 in detail. Our sample of five RGB members in the sparse nearby GC M30 (NGC 7099) were observed with the same HIRES configuration; all the resulting spectra are of high $\mathrm{S} / \mathrm{N}$. Table 1 gives details of the samples and spectra. Figures 1 and 2 show CMDs for the full Stetson online database (Stetson 2005) as well as our HIRES sample for each of these two GCs with $12 \mathrm{Gyr}$ isochrones from the Y2 grid (Kim et al. 2002) superposed. Figure 3 shows selected regions in the HIRES spectra of two stars from NGC 2419.

The determination of stellar parameters followed our earlier papers (see, e.g., Cohen \& Melendez 2005a, and references therein) and is based on $V-I, V-J$, and $V-K_{s}$ where the optical colors are from Stetson (2005) and the infrared colors are from the Two Micron All Sky Survey (2MASS; Skrutskie

5 MAKEE was developed by T.A. Barlow specifically for reduction of Keck HIRES data. It is freely available on the World Wide Web at the Keck Observatory home page, http://www2.keck.hawaii.edu/inst/hires/data_ reduction.html.

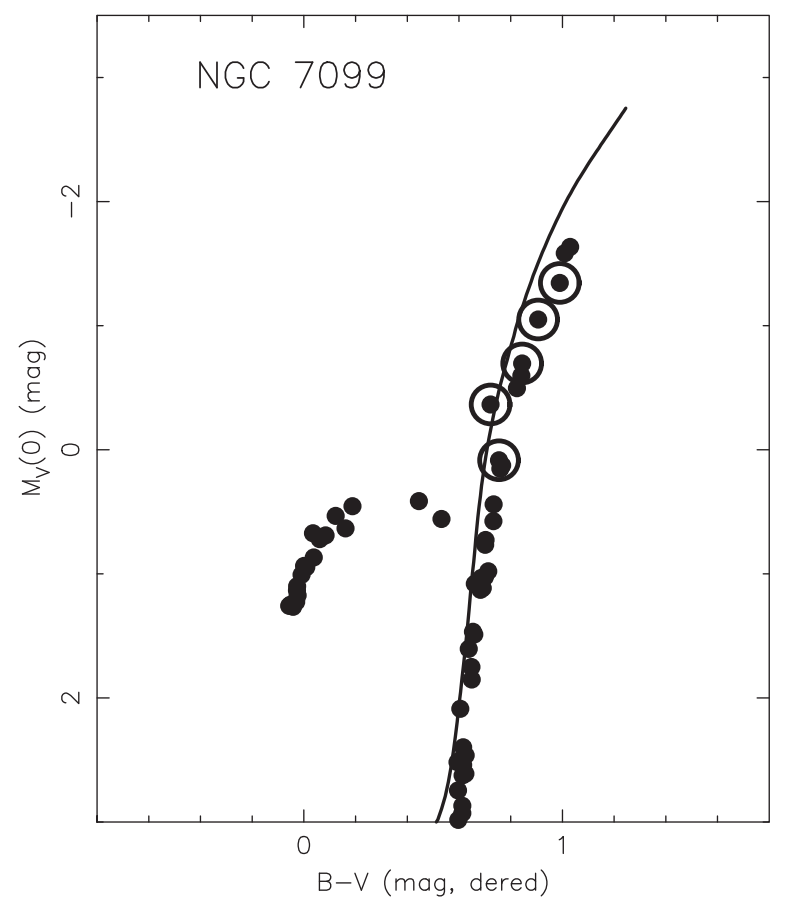

Figure 1. $M_{V}(0)-(B-V)_{0}$ CMD for NGC 7099 is shown from the Stetson (2005) online database. The RGB stars in our HIRES sample are circled. This is a sparse cluster, and there are few stars on the upper RGB. An $\alpha$-enhanced Y2 isochrone (Kim et al. 2002) with $[\mathrm{Fe} / \mathrm{H}]-2.2 \mathrm{dex}$ and age of $12 \mathrm{Gyr}$ is shown.

et al. 2006; Cutri et al. 2003). The uncertainties in 2MASS $K_{s}$ are rather large for the faint NGC 2419 giants, and only two of the NGC 7099 giants have an I mag in the Stetson (2005) database. 


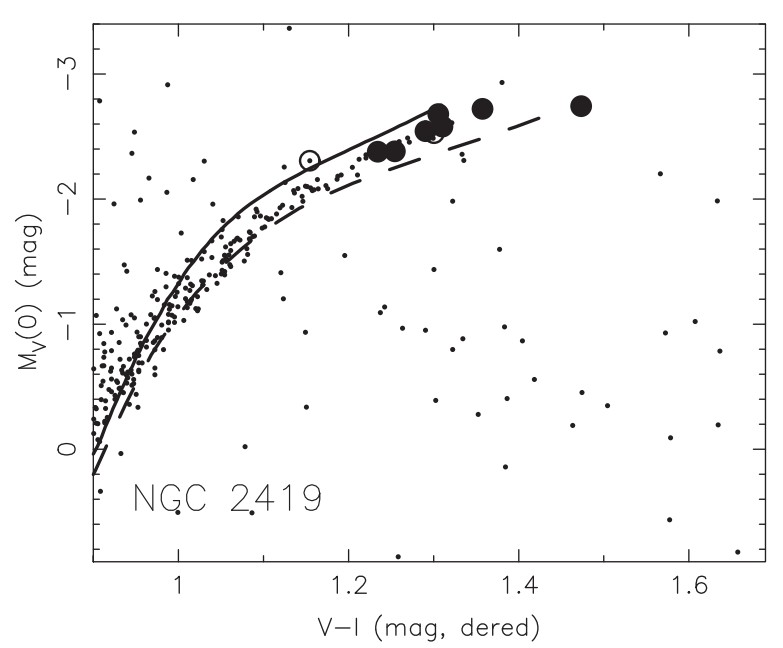

Figure 2. $M_{V}(0)-(V-I)_{0}$ CMD for the RGB in NGC 2419 is shown from the Stetson (2005) online database. The seven giants in our HIRES sample are indicated by large filled circles. Two other stars with lower S/N HIRES spectra, whose membership is questionable, are indicated by open circles. The two 12 Gyr Y2 isochrones (Kim et al. 2002) shown have $[\mathrm{Fe} / \mathrm{H}]-2.2$ and -1.9 dex, with $[\alpha / \mathrm{Fe}]+0.3$ dex.

We use the predicted color grid as a function of $T_{\text {eff }}, \log (g)$, and $[\mathrm{Fe} / \mathrm{H}]^{6}$ from Houdashelt et al. (2000). If we use the recent $T_{\text {eff }}$-color relations of Casagrande et al. (2010), which are not calibrated for such luminous giants, we obtain $T_{\text {eff }} \sim 35 \mathrm{~K}$ lower for the cooler luminosity stars in our sample for NGC 2419, ranging up to $105 \mathrm{~K}$ lower for the most luminous star in this GC. Alternative choices for $T_{\text {eff }}$ to be described below are given in Table 2. The surface gravities were calculated assuming a mass of $0.8 M_{\odot}$, the known distance, and the (low) interstellar extinction to each GC. The isochrone of the upper RGB for a very metal-poor old stellar system is such that an error of $100 \mathrm{~K}$ in $T_{\text {eff }}$ produces an error of 0.2 dex in the inferred surface gravity using this procedure.

The red giants in our sample in NGC 2419 span 0.36 mag in $V$ and a range of less than $200 \mathrm{~K}$ in $T_{\text {eff. The RGB stars in }}$ our sample for NGC 7099 are somewhat hotter in the mean due both to the somewhat lower metallicity of this GC and to the scarcity of upper RGB stars in this rather sparse cluster.

The detailed abundance analysis for each of the cluster giants follows J. Cohen's previous work (see, e.g., Cohen \& Melendez 2005a) using Kurucz (1993) LTE plane-parallel model stellar atmospheres and the analysis code MOOG (Sneden 1973). The adopted transition probabilities are largely from NIST version 3 . Note that the $g f$ values adopted here for the observed $\mathrm{Mg}$ I and $\mathrm{Ca}$ I lines have been updated from those we consistently used up to the present (see, e.g., Cohen \& Melendez 2005a) to match the current online values in the NIST version 4.0 (Ralchenko et al. 2010) database. The changes in abundance resulting from these updates are small, about -0.05 dex for $\mathrm{Mg}$ for a typical set of observed lines, and +0.03 dex for $\mathrm{Ca}$ I where more lines are usually observed, only some of which have $g f$ values that have been updated.

Hyperfine energy level splitting was used where appropriate, with many such patterns taken from Prochaska et al. (2000). The adopted damping constants are those described in Cohen \& Melendez (2005a).

\footnotetext{
6 The standard nomenclature is adopted; the abundance of element $\mathrm{X}$ is given by $\epsilon(\mathrm{X})=N(\mathrm{X}) / N(\mathrm{H})$ on a scale where $N(\mathrm{H})=10^{12} \mathrm{H}$ atoms. Then $[\mathrm{X} / \mathrm{H}]=\log [N(\mathrm{X}) / N(\mathrm{H})]-\log [N(\mathrm{X}) / N(\mathrm{H})]_{\odot}$ and similarly for $[\mathrm{X} / \mathrm{Fe}]$.
}

Table 2

Choice of $T_{\text {eff }}$ For Red Giant Members of NGC 2419

\begin{tabular}{lcccc}
\hline \hline Name $^{\mathrm{a}}$ & $\begin{array}{c}V^{\mathrm{a}} \\
(\mathrm{mag})\end{array}$ & $\begin{array}{c}T_{\text {eff }}(\text { Adopt }) \\
(\mathrm{K})\end{array}$ & $\begin{array}{c}T_{\text {eff }}(\text { Phot }), \sigma\left(T_{\text {eff }}\right)^{\mathrm{b}} \\
(\mathrm{K})\end{array}$ & $\begin{array}{c}\left.T_{\text {eff }} \text { (Isochrone }\right) \\
(\mathrm{K})\end{array}$ \\
\hline NGC 2419 & & & & \\
Stet 223 & 17.25 & 4250 & $4150(20)$ & 4264 \\
Stet 810 & 17.31 & 4330 & $4372(20)$ & 4286 \\
Stet 973 & 17.45 & 4320 & $4392(39)$ & 4340 \\
Stet 1131 & 17.61 & 4435 & $4428(35)^{\mathrm{c}}$ & 4384 \\
Stet 1209 & 17.41 & 4350 & $4328(46)$ & 4327 \\
Stet 1305 & 17.61 & 4400 & $4488(86)^{\mathrm{d}}$ & 4385 \\
Stet 1814 & 17.27 & 4370 & $4355(55)$ & 4274 \\
NGC 7099 & & & & $\ldots$ \\
S31 & 13.92 & 4790 & $4770(24)$ & $\ldots$ \\
S34 & 13.57 & 4658 & $4658(27)$ & $\ldots$ \\
AL38 & 13.27 & 4655 & $4655(52)^{\mathrm{e}}$ & $\ldots$ \\
S42 & 14.70 & 4935 & $4935(44)^{\mathrm{e}}$ & $\ldots$ \\
S81 & 14.25 & 5050 & $4970(64)^{\mathrm{e}}$ & $\ldots$ \\
\hline
\end{tabular}

Notes.

a Star IDs and $V$ mags are mostly from the online version of the database of Stetson (2005). See notes to Table 1 for details.

${ }^{\mathrm{b}} \mathrm{rms}$ of $T_{\mathrm{eff}}$ as derived from the available colors including $V-I, V-J$, and $V-K_{s}$.

c Uncertainty in 2MASS $K_{s}$ is 0.09 mag.

d Uncertainty in 2MASS $K_{s}$ is 0.10 mag.

e No I mag available.

Although it appears that small non-LTE corrections should be applied for several species, for example Na I (Takeda et al. 2003) and Ba II (Mashonkina et al. 2000), for consistency with our earlier work, the only non-LTE correction we have implemented is a fixed value of +0.60 dex for the resonance $\mathrm{Al}$ I doublet at 3944 and $3961 \AA$ following Baumuller \& Gehren (1996) (see also Andrievsky et al. 2008). Non-LTE for $\mathrm{K}_{\mathrm{I}}$ is discussed in Section 7.

Equivalent widths were measured in some cases by the script described in Section 3 of Cohen \& Melendez (2005a), which automatically searches for absorption features, fits a Gaussian, then matches those found to a master line list given the radial velocity of the star. For other sample stars, W. Huang used an IDL script to determine $W_{\lambda}$ for the set of lines in the master list. There was extensive hand checking of weak features, of most rare Earth lines, and of many of the strongest accepted lines. Lines with $W_{\lambda}>175 \mathrm{~m} \AA$ were rejected unless the species had only a few detected features. The resulting values, together with the atomic parameters for each line used, are given in Tables 3 and 4.

The $W_{\lambda}$ for the $3961 \AA \mathrm{Al}$ I line, when given, are particularly uncertain for the NGC 2419 giants; the $3944 \AA$ line was never used due to contamination by $\mathrm{CH}$. Both lines are uncomfortably far in the blue, where the $\mathrm{S} / \mathrm{N}$ of the HIRES spectra for such faint stars is degraded, but the $3961 \AA$ line is a key feature for this element as the $6690 \AA \mathrm{Al}$ I doublet is very weak and difficult to detect at the low metallicity of this GC.

Because of the uncertainty of the photometry for the NGC 2419 giants, we felt free to make small adjustments to the photometrically derived stellar parameters, primarily changing $T_{\text {eff }}$. These adjustments, detailed in Table 2, improved the excitation equilibrium for $\mathrm{Fe}$ I lines and the ionization balance between neutral and singly ionized lines of Fe and Ti. No such adjustment exceeded $100 \mathrm{~K}$ and was less than $50 \mathrm{~K}$ for four of the NGC 2419 giants. In the case of M30, only one star had $T_{\text {eff }}$ modified by more than $20 \mathrm{~K}$. For each star in NGC 2419, we 


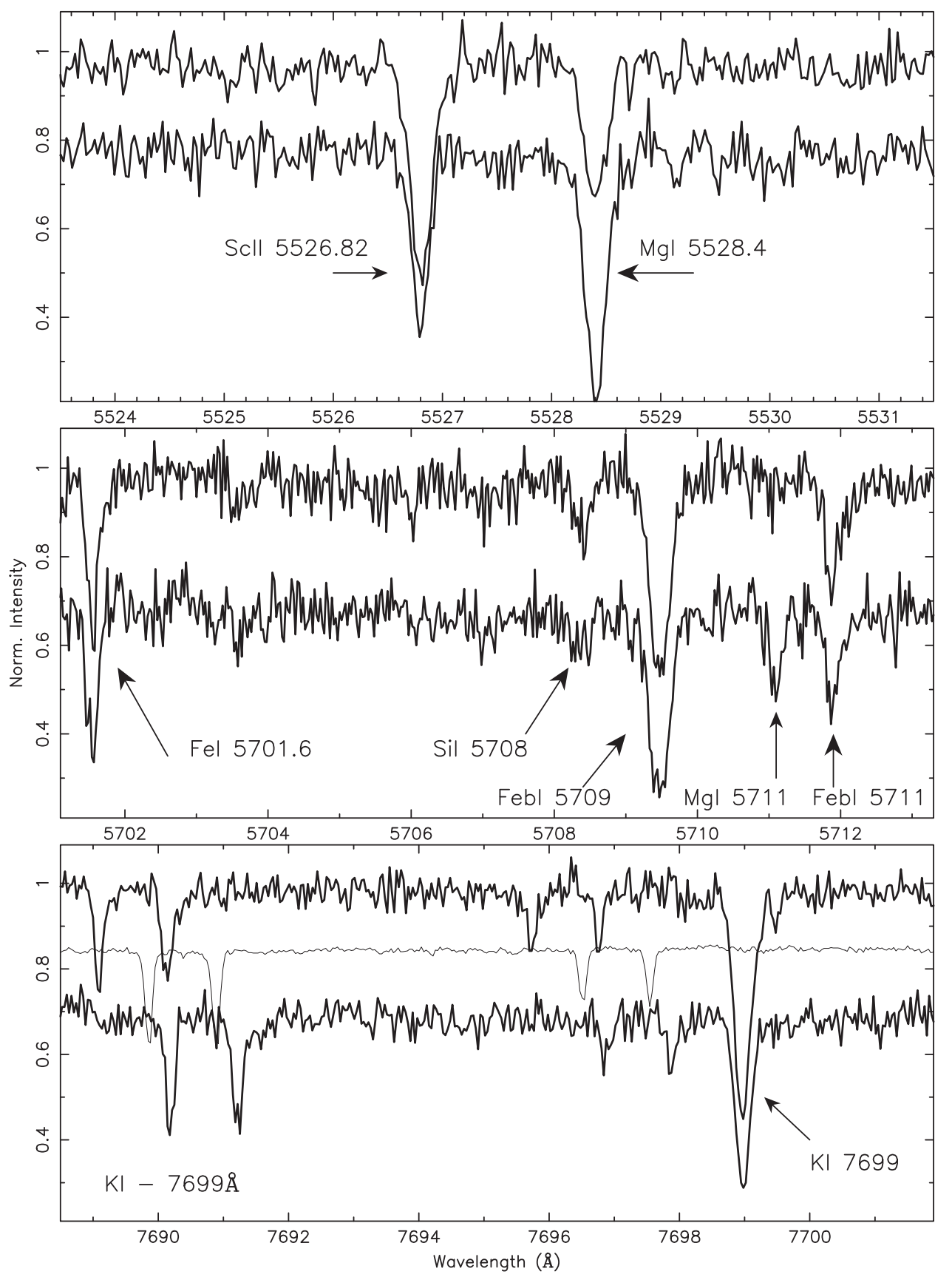

Figure 3. Regions of the spectrum near two $\mathrm{Mg}$ I lines and a line of $\mathrm{KI}$, shifted in wavelength into the rest frame, are shown for NGC 2419 S1131 as compared to $\mathrm{S} 1209$, whose spectrum is shifted slightly lower for clarity. These two giants differ in $V$ by 0.2 mag and in deduced $T_{\text {eff }}$ by less than $100 \mathrm{~K}$. The lines identified include a number of blends of Fe I lines or Fe I and Ni I lines labeled as "Febl." S1131 is more metal-rich and Mg-poor than S1209 and the other NGC 2419 giants. The thin line in the bottom panel is that of a rapidly rotating B star. The slight shifts in wavelength of the telluric lines between the spectra is a reflection of the differing heliocentric corrections for each spectrum.

measured 67 to $108 \mathrm{Fe}$ I lines, which allowed us to determine the microturbulent velocity directly from the spectra; every star in our sample in NGC 7099 had 99 or more detected Fe I lines.

Table 5 gives the slope of a linear fit to the Fe abundance deduced from each of the $\mathrm{Fe}$ I lines detected in a specific sample star as a function of excitation potential, reduced equivalent width $\left(W_{\lambda} / \lambda\right)$, and $\lambda$, where the first is most sensitive to the adopted $T_{\text {eff }}$, the second to $v_{t}$, and the third indicates any systematic problems in continuum opacity as a function of wavelength. Values are given for both GCs. In the ideal case, these slopes are zero. For NGC 2419 the typical range in $\chi$ for the $\mathrm{Fe}$ I lines is 0.9 to $4.2 \mathrm{eV}$; the stars with the best spectra span a larger range from 0.0 to $4.8 \mathrm{eV}$. The wavelength range for Fe I lines in most of the NGC 2419 (NGC 7099) spectra is $\sim 2400 \AA(\sim 3000 \AA)$, and a typical range in reduced equivalent width for such spectra is -5.4 to -4.5 dex for NGC 2419 and -6.3 to -4.4 dex for NGC 7099 . The crucial slope with $\chi$ ranged from -0.083 to $-0.038 \mathrm{dex} \mathrm{eV}^{-1}$ for the NGC 2419 sample and is $-0.048 \pm 0.007 \mathrm{dex} \mathrm{eV}^{-1}$ for the M30 RGB sample.

The resulting abundances for the seven RGB stars in NGC 2419 and the five in M30 are given in Tables 6 and 7, where all abundances are relative to Fe I. The adopted solar absolute abundances for each element can be inferred from the 
Table 3

Equivalent Widths for NGC 2419 RGB Stars

\begin{tabular}{|c|c|c|c|c|c|c|c|c|c|c|}
\hline $\begin{array}{l}\lambda \\
(\AA)\end{array}$ & Ion & $\begin{array}{l}\chi_{\text {exc }} \\
(\mathrm{eV})\end{array}$ & $\begin{array}{r}\log g f \\
(\operatorname{dex})\end{array}$ & $\begin{array}{l}\mathrm{S} 223 \\
(\mathrm{~m} \AA)\end{array}$ & $\begin{array}{c}\mathrm{S} 1814 \\
(\mathrm{~m} \AA)\end{array}$ & $\begin{array}{l}\text { S810 } \\
(\mathrm{m} \AA)\end{array}$ & $\begin{array}{c}\text { S1209 } \\
(\mathrm{m} \AA)\end{array}$ & $\begin{array}{l}\text { S973 } \\
(\mathrm{m} \AA)\end{array}$ & $\begin{array}{c}\mathrm{S} 1305 \\
(\mathrm{~m} \AA)\end{array}$ & $\begin{array}{l}\text { S1131 } \\
(\mathrm{m} \AA)\end{array}$ \\
\hline 6707.8 & Li I & 0.00 & 0.178 & $\ldots$ & $\ldots$ & $\ldots$ & $\ldots$ & $\ldots$ & $\ldots$ & $\leqslant 7.0$ \\
\hline 6300.30 & [O I] & 0.00 & -9.780 & $\ldots$ & $\ldots$ & $\ldots$ & 36.7 & 54.7 & $\ldots$ & $\ldots$ \\
\hline 6363.78 & [OI] & 0.02 & -10.300 & $\ldots$ & $\ldots$ & $\ldots$ & $\ldots$ & 23.5 & $\ldots$ & $\ldots$ \\
\hline 5682.63 & $\mathrm{NaI}$ & 2.10 & -0.700 & 30.3 & 14.9 & 6.0 & $\ldots$ & $\ldots$ & $\ldots$ & 13.0 \\
\hline 5688.19 & $\mathrm{NaI}$ & 2.10 & -0.420 & 46.0 & $\ldots$ & 15.0 & $\ldots$ & $\ldots$ & $\ldots$ & 36.0 \\
\hline 5889.95 & $\mathrm{NaI}$ & 0.00 & 0.110 & $\ldots$ & $\ldots$ & $\ldots$ & $\ldots$ & 362.0 & 376.0 & $\ldots$ \\
\hline 5895.92 & $\mathrm{NaI}$ & 0.00 & -0.190 & $\ldots$ & $\ldots$ & 236.0 & 282.0 & 279.0 & 355.0 & $\ldots$ \\
\hline 4703.00 & $\mathrm{Mg}_{\mathrm{I}}$ & 4.34 & -0.440 & 92.0 & 140.0 & 96.4 & 125.0 & 123.0 & 102.3 & 56.0 \\
\hline 5172.70 & $\mathrm{Mg}_{\mathrm{I}}$ & 2.71 & -0.380 & 340.0 & 365.0 & $\ldots$ & $\ldots$ & $\ldots$ & 312.2 & 224.0 \\
\hline 5183.62 & $\mathrm{Mg}_{\mathrm{I}}$ & 2.72 & -0.160 & 406.8 & 419.9 & $\ldots$ & $\ldots$ & $\ldots$ & 365.6 & 266.0 \\
\hline 5528.40 & $\mathrm{Mg}_{\mathrm{I}}$ & 4.34 & -0.498 & 158.5 & 150.0 & 138.7 & 139.2 & 122.2 & 120.9 & 71.0 \\
\hline 5711.09 & $\mathrm{Mg}_{\mathrm{I}}$ & 4.34 & -1.724 & 44.0 & 48.0 & 47.5 & 43.3 & 73.0 & 40.8 & $\leqslant 15.5$ \\
\hline 3961.52 & $\mathrm{Al} I$ & 0.00 & -0.340 & 226.1 & 228.0 & 121.5 & 203.6 & $\ldots$ & $\ldots$ & 240.5 \\
\hline 6696.02 & $\mathrm{Al}$ I & 3.14 & -1.340 & 15.7 & 11.5 & 7.5 & 5.0 & $\ldots$ & $\ldots$ & 10.9 \\
\hline 4102.94 & Si I & 1.91 & -3.140 & $\ldots$ & $\ldots$ & $\ldots$ & $\ldots$ & $\ldots$ & $\ldots$ & 150.8 \\
\hline
\end{tabular}

(This table is available in its entirety in a machine-readable form in the online journal. A portion is shown here for guidance regarding its form and content.)

Table 4

Equivalent Widths For NGC 7099 RGB Stars

\begin{tabular}{|c|c|c|c|c|c|c|c|c|}
\hline $\begin{array}{l}\lambda \\
(\AA)\end{array}$ & Ion & $\begin{array}{l}\chi_{\text {exc }} \\
(\mathrm{eV})\end{array}$ & $\begin{array}{r}\log g f \\
(\operatorname{dex})\end{array}$ & $\begin{array}{c}\text { S31 } \\
(\mathrm{m} \AA)\end{array}$ & $\begin{array}{c}\mathrm{S} 34 \\
(\mathrm{~m} \AA)\end{array}$ & $\begin{array}{c}\mathrm{S} 38 \\
(\mathrm{~m} \AA)\end{array}$ & $\begin{array}{c}\mathrm{S} 42 \\
(\mathrm{~m} \AA)\end{array}$ & $\begin{array}{c}\mathrm{S} 81 \\
(\mathrm{~m} \AA)\end{array}$ \\
\hline 6300.30 & [OI] & 0.00 & -9.780 & 8.0 & 8.7 & 7.8 & 7.8 & 5.0 \\
\hline 6363.78 & [OI] & 0.02 & -10.300 & 3.4 & $\ldots$ & 3.3 & $\ldots$ & 6.0 \\
\hline 7771.94 & $\mathrm{O}_{\mathrm{I}}$ & 9.15 & 0.369 & 6.5 & 3.7 & 4.2 & 7.9 & 4.3 \\
\hline 7774.17 & OI & 9.15 & 0.223 & $\ldots$ & $\ldots$ & $\ldots$ & 7.5 & $\ldots$ \\
\hline 5682.63 & $\mathrm{NaI}$ & 2.10 & -0.700 & $\ldots$ & 9.0 & 12.2 & $\ldots$ & 10.0 \\
\hline 5688.19 & $\mathrm{NaI}$ & 2.10 & -0.420 & 11.1 & 15.0 & 21.6 & $\ldots$ & 15.0 \\
\hline 5889.95 & $\mathrm{NaI}$ & 0.00 & 0.110 & 217.1 & 236.6 & 261.9 & 171.0 & 239.0 \\
\hline 5895.92 & $\mathrm{NaI}$ & 0.00 & -0.190 & 195.9 & 210.8 & 232.7 & 157.3 & 215.2 \\
\hline 6160.75 & $\mathrm{NaI}$ & 2.00 & -1.230 & 4.0 & $\ldots$ & $\ldots$ & $\ldots$ & $\ldots$ \\
\hline 4057.52 & $M g_{I}$ & 4.34 & -1.200 & 65.2 & 67.3 & 47.3 & 51.5 & 41.3 \\
\hline 4167.28 & $\mathrm{Mg}_{\mathrm{I}}$ & 4.34 & -0.745 & 79.6 & 88.5 & 66.9 & 69.6 & 48.0 \\
\hline 4703.00 & $M g_{I}$ & 4.34 & -0.440 & 89.4 & 96.5 & 84.8 & 77.9 & 57.8 \\
\hline 5172.70 & $\mathrm{Mg}_{\mathrm{I}}$ & 2.71 & -0.380 & 235.0 & 295.0 & 234.9 & 205.9 & 183.4 \\
\hline 5183.62 & $\mathrm{Mg}_{\mathrm{I}}$ & 2.72 & -0.160 & 267.5 & 328.0 & 264.6 & 237.9 & 204.4 \\
\hline 5528.40 & $M g_{I}$ & 4.34 & -0.498 & 100.5 & 105.0 & 99.6 & 83.5 & 68.5 \\
\hline
\end{tabular}

(This table is available in its entirety in a machine-readable form in the online journal. A portion is shown here for guidance regarding its form and content.)

entries in Table 7. With regard to the ionization equilibrium that for $\mathrm{Fe}$ I versus $\mathrm{Fe}$ II is generally quite good, within 0.1 dex, for all stars in both NGC 2419 and in M30. The ionization equilibrium of Ti I versus Ti II is somewhat worse, typically within $\sim 0.2$ dex, with the Ti II lines giving a somewhat higher Ti abundance.

Tables 8 and 9 give the sensitivity of the derived absolute abundances $[\mathrm{X} / \mathrm{H}]$ and those relative to $\mathrm{Fe}[\mathrm{X} / \mathrm{Fe}]$ for small variations in the various relevant stellar and analysis parameters.

\section{ALTERNATIVE CHOICES OF STELLAR PARAMETERS}

The choice of stellar parameters is crucial for determining absolute stellar abundances, although less critical for abundance ratios $[\mathrm{X} / \mathrm{Fe}]$ as many of the systematic effects cancel, as is shown by comparing the entries for each species in Table 8 with those in Table 9. We have therefore explored several methods of determining $T_{\text {eff }}$. We call the $T_{\text {eff }}$ determined by the mean of that inferred from each available color $(V-I, V-J$, and $V-K_{s}$ ), the photometric $T_{\text {eff }}$. The value of $T_{\text {eff }}$ adopted for the abundance analysis, $T_{\text {eff }}$ (adopt), is initially set to $T_{\text {eff }}$ (phot),
Table 5

Deduced Slopes for Fe I Line-by-Line Abundances

\begin{tabular}{lcrr}
\hline \hline ID & $\begin{array}{c}\text { FeI vs. EP } \\
\left(\operatorname{dex~eV}^{-1}\right)\end{array}$ & $\begin{array}{c}\text { Fe I vs. }\left(W_{\lambda} / \lambda\right) \\
(\operatorname{dex})\end{array}$ & \multicolumn{1}{c}{$\begin{array}{c}\text { Fe I vs. } \lambda \\
\left(\operatorname{dex} \AA^{-1}\right)\end{array}$} \\
\hline NGC 2419 & & & \\
S1131 & -0.042 & 0.010 & $-0.118 \mathrm{e}-4$ \\
S1305 & -0.046 & -0.075 & $0.168 \mathrm{e}-4$ \\
S1209 & -0.050 & -0.001 & $0.451 \mathrm{e}-4$ \\
S973 & -0.038 & 0.045 & $0.093 \mathrm{e}-4$ \\
S1814 & -0.083 & -0.001 & $0.169 \mathrm{e}-4$ \\
S223 & -0.079 & 0.077 & $-0.136 \mathrm{e}-4$ \\
NGC 7099 & & & \\
S31 & -0.041 & 0.008 & $-0.022 \mathrm{e}-4$ \\
S34 & -0.042 & 0.008 & $-0.173 \mathrm{e}-4$ \\
S38 & -0.042 & 0.017 & $-0.153 \mathrm{e}-4$ \\
S42 & -0.055 & 0.012 & $-0.031 \mathrm{e}-4$ \\
S81 & -0.047 & 0.009 & $-0.087 \mathrm{e}-5$ \\
\hline
\end{tabular}

with small adjustments permitted to improve the $\mathrm{Fe}$ I slopes and the ionization equilibrium as described above. 
Table 6

Abundances for Red Giant Members of NGC 2419

\begin{tabular}{|c|c|c|c|c|c|c|c|c|c|c|c|c|c|c|c|c|c|c|c|c|c|}
\hline $\begin{array}{l}\text { ID }^{\mathrm{a}} \\
\text { Species }\end{array}$ & $\begin{array}{c}\mathrm{S} 1131 \\
{[\mathrm{X} / \mathrm{Fe}]} \\
(\mathrm{dex})\end{array}$ & $\begin{array}{c}\sigma^{b} \\
(\operatorname{dex})\end{array}$ & $N^{\mathrm{c}}$ & $\begin{array}{c}\mathrm{S} 1305 \\
{[\mathrm{X} / \mathrm{Fe}]} \\
(\mathrm{dex})\end{array}$ & $\begin{array}{c}\sigma^{\mathrm{b}} \\
(\mathrm{dex})\end{array}$ & $N$ & $\begin{array}{c}\mathrm{S} 1209 \\
{[\mathrm{X} / \mathrm{Fe}]} \\
(\mathrm{dex})\end{array}$ & $\begin{array}{c}\sigma^{\mathrm{b}} \\
(\operatorname{dex})\end{array}$ & $N$ & $\begin{array}{c}\text { S973 } \\
{[\mathrm{X} / \mathrm{Fe}]} \\
(\mathrm{dex})\end{array}$ & $\begin{array}{c}\sigma^{\mathrm{b}} \\
(\mathrm{dex})\end{array}$ & $N$ & $\begin{array}{c}\mathrm{S} 810 \\
{[\mathrm{X} / \mathrm{Fe}]} \\
(\mathrm{dex})\end{array}$ & $\begin{array}{c}\sigma^{\mathrm{b}} \\
(\mathrm{dex})\end{array}$ & $N$ & $\begin{array}{c}\mathrm{S} 1814 \\
{[\mathrm{X} / \mathrm{Fe}]} \\
(\mathrm{dex})\end{array}$ & $\begin{array}{c}\sigma^{b} \\
(\operatorname{dex})\end{array}$ & $N$ & $\begin{array}{c}\mathrm{S} 223 \\
{[\mathrm{X} / \mathrm{Fe}]} \\
(\mathrm{dex})\end{array}$ & $\begin{array}{c}\sigma^{\mathrm{b}} \\
(\mathrm{dex})\end{array}$ & $N$ \\
\hline $\mathrm{C}^{\mathrm{d}}$ & -0.81 & $\ldots$ & 1 & -0.26 & 0.30 & 1 & -0.73 & $\ldots$ & 1 & $\ldots$ & $\ldots$ & $\ldots$ & -0.90 & $\ldots$ & 1 & -0.79 & $\ldots$ & 1 & $\ldots$ & $\ldots$ & $\ldots$ \\
\hline OI & $\ldots$ & $\ldots$ & $\ldots$ & $\ldots$ & $\ldots$ & $\ldots$ & 0.67 & $\ldots$ & 1 & 0.71 & 0.03 & 2 & $\ldots$ & $\ldots$ & $\ldots$ & $\ldots$ & $\ldots$ & $\ldots$ & $\ldots$ & $\ldots$ & $\ldots$ \\
\hline $\mathrm{Na} \mathrm{I}$ & -0.04 & 0.19 & 2 & 0.54 & 0.15 & 2 & 0.23 & $\ldots$ & 1 & 0.06 & 0.13 & 2 & -0.42 & 0.09 & 3 & -0.04 & . & 1 & 0.29 & 0.01 & 2 \\
\hline Mg I & -0.47 & 0.17 & $5^{\mathrm{e}}$ & 0.32 & 0.15 & 5 & 0.62 & 0.18 & 3 & 0.46 & 0.20 & 3 & 0.32 & 0.22 & 3 & 0.55 & 0.19 & 5 & 0.30 & 0.22 & 5 \\
\hline $\mathrm{Al} \mathrm{I}^{\mathrm{f}}$ & 0.45 & $\ldots$ & 1 & $\ldots$ & $\ldots$ & $\ldots$ & $\ldots$ & $\ldots$ & $\ldots$ & $\ldots$ & $\ldots$ & $\ldots$ & $\ldots$ & $\ldots$ & $\cdots$ & $\ldots$ & $\ldots$ & $\ldots$ & $\ldots$ & $\ldots$ & $\ldots$ \\
\hline Si I & 0.56 & 0.16 & 13 & 0.54 & 0.18 & 4 & $\ldots$ & $\ldots$ & $\ldots$ & 0.43 & 0.32 & 4 & $\ldots$ & & $\ldots$ & 0.33 & 0.17 & 5 & 0.30 & 0.17 & 4 \\
\hline $\mathrm{K}_{\mathrm{I}}$ & 1.13 & $\ldots$ & 1 & 0.36 & $\ldots$ & 1 & 0.47 & $\ldots$ & 1 & $\ldots$ & $\ldots$ & $\ldots$ & 0.45 & $\ldots$ & 1 & 0.49 & $\ldots$ & 1 & 0.55 & $\ldots$ & 1 \\
\hline $\mathrm{Ca} \mathrm{I}$ & 0.23 & 0.18 & 18 & 0.21 & 0.22 & 15 & 0.20 & 0.14 & 14 & 0.17 & 0.22 & 15 & 0.05 & 0.14 & 11 & 0.12 & 0.17 & 18 & 0.08 & 0.16 & 14 \\
\hline Sc II & 0.32 & 0.08 & 7 & 0.15 & 0.20 & 7 & 0.08 & 0.13 & 6 & -0.02 & 0.16 & 7 & -0.01 & 0.08 & 7 & 0.08 & 0.12 & 8 & 0.19 & 0.07 & 7 \\
\hline Ti I & 0.16 & 0.17 & 23 & 0.18 & 0.19 & 10 & 0.06 & 0.15 & 17 & 0.08 & 0.21 & 9 & 0.00 & 0.17 & 14 & 0.15 & 0.16 & 23 & 0.17 & 0.13 & 19 \\
\hline Ti II & 0.34 & 0.33 & 9 & 0.28 & 0.09 & 4 & 0.38 & 0.16 & 10 & 0.08 & 0.16 & 3 & 0.02 & 0.27 & 7 & 0.40 & 0.14 & 7 & 0.25 & 0.27 & 5 \\
\hline V I & 0.03 & 0.15 & 4 & 0.18 & 0.43 & 2 & 0.08 & 0.13 & 3 & 0.01 & 0.07 & 3 & 0.03 & 0.07 & 3 & -0.08 & 0.11 & 4 & -0.07 & 0.14 & 9 \\
\hline CrI & -0.08 & 0.22 & 7 & -0.37 & 0.25 & 2 & -0.23 & 0.13 & 6 & -0.25 & 0.03 & 2 & -0.31 & 0.08 & 4 & -0.20 & 0.14 & 6 & -0.18 & 0.16 & 5 \\
\hline Mn I & -0.24 & 0.16 & 7 & -0.28 & 0.09 & 5 & -0.27 & 0.18 & 6 & -0.18 & 0.24 & 4 & -0.39 & 0.12 & 6 & -0.25 & 0.09 & 6 & -0.33 & 0.11 & 6 \\
\hline $\mathrm{Fe}^{\mathrm{g}}$ & -1.95 & 0.15 & 108 & -2.17 & 0.18 & 71 & -2.20 & 0.10 & 75 & -1.98 & 0.17 & 67 & -2.00 & 0.12 & 78 & -2.04 & 0.14 & 93 & -2.09 & 0.15 & 91 \\
\hline Fe II & -0.12 & 0.21 & 11 & -0.05 & 0.17 & 8 & -0.01 & 0.21 & 9 & -0.11 & 0.26 & 6 & -0.01 & 0.15 & 6 & -0.08 & 0.20 & 14 & -0.02 & 0.20 & 12 \\
\hline Co I & 0.03 & $\ldots$ & 1 & $\ldots$ & $\ldots$ & $\ldots$ & 0.04 & $\ldots$ & 1 & $\ldots$ & $\ldots$ & $\ldots$ & 0.21 & 0.18 & 3 & $\ldots$ & $\ldots$ & $\ldots$ & 0.10 & 0.01 & 2 \\
\hline Ni I & -0.04 & 0.19 & 18 & -0.05 & 0.22 & 13 & -0.08 & 0.15 & 11 & -0.08 & 0.25 & 14 & 0.02 & 0.26 & 13 & -0.07 & 0.20 & 21 & -0.07 & 0.18 & 21 \\
\hline $\mathrm{Cu} \mathrm{I}$ & -0.65 & $\ldots$ & 1 & -0.55 & $\ldots$ & 1 & -0.63 & $\ldots$ & 1 & -0.48 & $\ldots$ & 1 & -0.63 & $\ldots$ & 1 & -0.52 & $\ldots$ & 1 & -0.79 & 0.02 & 2 \\
\hline $\mathrm{ZnI}$ & -0.14 & 0.13 & 2 & $\ldots$ & $\ldots$ & $\ldots$ & $\ldots$ & $\ldots$ & $\ldots$ & $\ldots$ & $\ldots$ & $\ldots$ & -0.23 & 0.02 & 2 & -0.16 & 0.30 & 2 & -0.27 & 0.18 & 2 \\
\hline Sr I & -0.30 & $\ldots$ & 1 & $\ldots$ & $\ldots$ & $\ldots$ & -0.31 & $\ldots$ & 1 & $\ldots$ & $\ldots$ & $\ldots$ & -0.28 & $\ldots$ & 1 & -0.04 & $\ldots$ & 1 & $\ldots$ & $\ldots$ & $\ldots$ \\
\hline Sr II & $\ldots$ & $\ldots$ & $\ldots$ & $\ldots$ & $\ldots$ & $\ldots$ & -0.18 & $\ldots$ & 1 & $\ldots$ & $\ldots$ & $\ldots$ & -0.15 & $\ldots$ & 1 & -0.02 & $\ldots$ & 1 & $\ldots$ & $\ldots$ & $\ldots$ \\
\hline Y II & -0.46 & 0.09 & 3 & -0.42 & 0.13 & 3 & -0.37 & 0.10 & 5 & -0.43 & 0.07 & 2 & -0.56 & 0.21 & 4 & -0.43 & 0.15 & 4 & -0.27 & 0.30 & 4 \\
\hline Zr I & $\ldots$ & $\ldots$ & $\ldots$ & $\ldots$ & $\ldots$ & $\ldots$ & $\ldots$ & $\ldots$ & $\ldots$ & $\ldots$ & $\ldots$ & $\ldots$ & . & $\ldots$ & $\ldots$ & $\ldots$ & $\ldots$ & $\ldots$ & 0.45 & $\ldots$ & 1 \\
\hline Ba II & -0.18 & 0.05 & 4 & -0.03 & 0.05 & 3 & -0.08 & 0.13 & 3 & -0.22 & 0.16 & 3 & -0.41 & 0.13 & 3 & -0.25 & 0.11 & 3 & -0.01 & 0.23 & 3 \\
\hline La II & -0.09 & $\ldots$ & 1 & $\ldots$ & $\ldots$ & $\ldots$ & 0.38 & $\ldots$ & 1 & $\ldots$ & $\ldots$ & $\ldots$ & -0.34 & $\ldots$ & 1 & 0.05 & $\ldots$ & 1 & $\ldots$ & $\ldots$ & $\ldots$ \\
\hline Ce II & -0.17 & 0.14 & 3 & $\ldots$ & $\ldots$ & $\ldots$ & -0.09 & 0.06 & 2 & $\ldots$ & $\ldots$ & $\ldots$ & -0.30 & 0.24 & 3 & -0.52 & 0.09 & 2 & $\ldots$ & $\ldots$ & $\ldots$ \\
\hline $\mathrm{Nd}$ II & -0.18 & 0.20 & 5 & 0.37 & $\ldots$ & 1 & 0.03 & 0.13 & 2 & $\leqslant 0.05$ & $\ldots$ & 1 & -0.18 & 0.04 & 2 & -0.06 & 0.08 & 3 & -0.26 & 0.17 & 4 \\
\hline Eu II & 0.31 & 0.28 & 2 & $\leqslant 0.77$ & $\ldots$ & 1 & 0.39 & 0.11 & 2 & $\ldots$ & $\ldots$ & $\ldots$ & 0.04 & 0.20 & 2 & 0.21 & 0.11 & 2 & 0.30 & 0.16 & 2 \\
\hline Dy II & $\ldots$ & $\ldots$ & $\ldots$ & $\ldots$ & $\ldots$ & $\ldots$ & $\ldots$ & $\ldots$ & $\ldots$ & $\ldots$ & $\ldots$ & $\ldots$ & $\ldots$ & $\ldots$ & $\ldots$ & 0.37 & $\ldots$ & 1 & $\ldots$ & $\ldots$ & $\ldots$ \\
\hline
\end{tabular}

Notes.

a Star IDs from online version of Stetson (2005).

${ }^{\mathrm{b}}$ Dispersion about the mean abundance.

c Number of detected lines.

d Synthesis of $\mathrm{CH}$ band near $4300 \AA$.

e Upper limit for $5711 \AA \mathrm{Mg}$ I line treated as a detection.

f Includes a +0.6 dex non-LTE correction for the $3961 \AA$ line.

$\mathrm{g}[\mathrm{Fe} / \mathrm{H}]$ from Fe I lines. All $[\mathrm{X} / \mathrm{Fe}]$ refer to $[\mathrm{Fe} / \mathrm{H}]$ from $\mathrm{Fe}$. The same solar abundances as are used as in Table 7.

Another $T_{\text {eff }}$ can be determined by assuming that all the stars are RGB members of their cluster, and that all the cluster stars have the same initial chemical inventory and age. Hence they must lie along a single old very metal-poor isochrone. Here we are using a single magnitude, chosen on the basis of its high measurement accuracy and discrimination along the isochrone, to determine $T_{\text {eff }}$ (iso). This removes the issue of random errors in colors, which is of particular concern for NGC 2419, with its large distance and hence faint RGB stars near the limit for 2MASS photometry. With a large enough sample, the "isochrone" itself can be defined from the mean locus of the stars in the cluster CMD, as was done in Cohen \& Melendez (2005a); see also the large Very Large Telescope (VLT) study of Carretta et al. (2010) and references therein. In the present case, even if the HIRES sample in NGC 2419 were much larger, the concern that this particular GC may not be chemically uniform would limit the applicability of such an approach. If the isochrone chosen is appropriate for the cluster, then the mean $\Delta\left[T_{\text {eff }}\right.$ (phot) $-T_{\text {eff }}$ (iso) $]$ will be zero. If the isochrone is close, but not exactly that of the cluster, then the mean difference will be a constant.
Table 2 gives the values of the adopted, photometric, and isochrone $T_{\text {eff }}$ for the sample of RGB stars in NGC 2419. The rms $\sigma$ about the mean for the set of available colors from the three we consider is given for the $T_{\text {eff }}$ (phot) in parentheses. The photometric $T_{\text {eff }}$ was adopted for the detailed abundance analysis for three of the five stars in NGC 7099. For the other two, the differences are $18 \mathrm{~K}(0.8 \sigma)$ for S31 and $79 \mathrm{~K}(1.2 \sigma)$ for S34. The isochrone and photometric values of $T_{\text {eff }}$ for this nearby GC are essentially identical. For NGC 2419 that is not the case. The brightest star (S223) at the RGB tip is somewhat redder than would be expected from the isochrone.

\section{INNER VERSUS OUTER HALO}

A comparison of the mean abundance ratios in the two GCs discussed here M30 (NGC 7099), at a distance of only $8 \mathrm{kpc}$, with the very distant outer halo globular cluster NGC 2419 is given in Table 10 and illustrated in detail in Figure 4. There is no substantive difference between the elemental abundance ratios for luminous red giants in the outer halo cluster NGC 2419 and the ratios of inner halo clusters. The inner halo abundance 
Table 7

Abundances for Red Giant Members of NGC 7099

\begin{tabular}{|c|c|c|c|c|c|c|c|c|c|c|c|c|c|c|c|c|}
\hline $\begin{array}{l}\mathrm{ID}^{\mathrm{a}} \\
\text { Species }\end{array}$ & $\begin{array}{c}\text { Stet } \\
{[\mathrm{X} / \mathrm{Fe}]} \\
(\mathrm{dex})\end{array}$ & $\begin{array}{c}31 \\
\log [\epsilon(X)] \\
(\operatorname{dex})\end{array}$ & $N^{\mathrm{b}}$ & $\begin{array}{c}\sigma^{\mathrm{c}} \\
(\operatorname{dex})\end{array}$ & $\begin{array}{c}\text { Stet } \\
\log [\epsilon(X)] \\
(\operatorname{dex})\end{array}$ & $\begin{array}{l}34 \\
N^{\mathrm{b}}\end{array}$ & $\begin{array}{c}\sigma^{c} \\
(\operatorname{dex})\end{array}$ & $\begin{array}{c}\text { AL } \\
\log [\epsilon(X)] \\
(\operatorname{dex})\end{array}$ & $\begin{array}{l}38 \\
N^{\mathrm{b}}\end{array}$ & $\begin{array}{c}\sigma^{\mathrm{c}} \\
(\mathrm{dex})\end{array}$ & $\begin{array}{c}\text { Stet } \\
\log [\epsilon(\mathrm{X})] \\
(\operatorname{dex})\end{array}$ & $\begin{array}{l}42 \\
N^{\mathrm{b}}\end{array}$ & $\begin{array}{c}\sigma^{\mathrm{c}} \\
(\mathrm{dex})\end{array}$ & $\begin{array}{c}\text { Stet } \\
\log [\epsilon(X)] \\
(\operatorname{dex})\end{array}$ & $\begin{array}{l}81 \\
N^{\mathrm{b}}\end{array}$ & $\begin{array}{c}\sigma^{\mathrm{c}} \\
(\operatorname{dex})\end{array}$ \\
\hline$C^{d}$ & -0.11 & 6.09 & 1 & $\ldots$ & 5.76 & 1 & $\ldots$ & 5.48 & 1 & $\ldots$ & 6.36 & 1 & $\ldots$ & 5.73 & 1 & $\ldots$ \\
\hline $\mathrm{OI}_{\mathrm{I}}$ & 0.76 & 7.20 & 3 & 0.08 & 6.86 & 2 & 0.21 & 6.91 & 3 & 0.17 & 7.26 & 3 & 0.13 & 6.65 & 1 & $\ldots$ \\
\hline $\mathrm{Na} \mathrm{I}$ & 0.20 & 4.13 & 4 & 0.10 & 4.18 & 4 & 0.09 & 4.33 & 4 & 0.14 & 3.89 & 2 & 0.11 & 4.49 & 4 & 0.22 \\
\hline $\mathrm{Mg}_{\mathrm{I}}$ & 0.53 & 5.68 & 7 & 0.17 & 5.82 & 7 & 0.12 & 5.53 & 7 & 0.08 & 5.57 & 7 & 0.20 & 5.41 & 7 & 0.17 \\
\hline $\mathrm{Al} \mathrm{I}{ }^{\mathrm{e}}$ & 0.21 & 4.29 & 1 & $\ldots$ & 4.75 & 1 & $\ldots$ & 4.82 & 1 & $\ldots$ & 4.07 & 1 & $\ldots$ & 4.85 & 2 & 0.11 \\
\hline Si I & 0.46 & 5.62 & 8 & 0.11 & 5.69 & 9 & 0.10 & 5.64 & 9 & 0.10 & 5.62 & 4 & 0.18 & 5.69 & 6 & 0.12 \\
\hline $\mathrm{K}_{\mathrm{I}}$ & 0.57 & 3.30 & 1 & $\ldots$ & 3.48 & 1 & $\ldots$ & 3.19 & 1 & $\ldots$ & 3.15 & 1 & $\ldots$ & 3.39 & 1 & $\ldots$ \\
\hline $\mathrm{Ca} \mathrm{I}$ & 0.28 & 4.25 & 21 & 0.16 & 4.31 & 23 & 0.15 & 4.15 & 21 & 0.15 & 4.24 & 20 & 0.18 & 4.20 & 20 & 0.14 \\
\hline Sc II & 0.23 & 0.94 & 10 & 0.10 & 0.91 & 10 & 0.11 & 0.88 & 10 & 0.08 & 0.89 & 7 & 0.14 & 0.87 & 8 & 0.13 \\
\hline Ti I & 0.21 & 2.81 & 22 & 0.10 & 2.84 & 18 & 0.06 & 2.68 & 18 & 0.09 & 2.76 & 17 & 0.09 & 2.79 & 17 & 0.05 \\
\hline Ti II & 0.41 & 3.01 & 22 & 0.13 & 2.97 & 20 & 0.14 & 2.97 & 20 & 0.15 & 2.99 & 21 & 0.13 & 2.87 & 24 & 0.15 \\
\hline V I & -0.22 & 1.39 & 1 & $\ldots$ & 1.59 & 3 & 0.06 & 1.20 & 1 & $\ldots$ & 1.27 & 1 & $\ldots$ & 1.28 & 1 & $\ldots$ \\
\hline CrI & -0.32 & 2.96 & 9 & 0.09 & 3.07 & 10 & 0.11 & 2.88 & 10 & 0.13 & 2.95 & 10 & 0.08 & 2.88 & 10 & 0.12 \\
\hline Mn I & -0.39 & 2.61 & 6 & 0.06 & 2.57 & 7 & 0.05 & 2.59 & 7 & 0.08 & 2.56 & 6 & 0.13 & 2.50 & 6 & 0.13 \\
\hline $\mathrm{Fe} \mathrm{I}^{\mathrm{f}}$ & -2.39 & 5.06 & 121 & 0.13 & 5.13 & 125 & 0.14 & 4.97 & 115 & 0.14 & 4.99 & 99 & 0.15 & 4.97 & 107 & 0.13 \\
\hline Fe II & 0.05 & 5.11 & 16 & 0.14 & 5.11 & 20 & 0.11 & 5.09 & 20 & 0.13 & 5.05 & 15 & 0.19 & 5.01 & 16 & 0.14 \\
\hline Co I & -0.26 & 2.27 & 1 & $\ldots$ & 2.29 & 2 & 0.05 & 2.33 & 2 & 0.16 & 2.39 & 1 & $\ldots$ & 2.42 & 1 & $\ldots$ \\
\hline Ni I & 0.02 & 3.88 & 14 & 0.18 & 3.87 & 16 & 0.18 & 3.77 & 16 & 0.16 & 3.86 & 9 & 0.23 & 3.86 & 6 & 0.19 \\
\hline $\mathrm{Cu} \mathrm{I}$ & -0.77 & 1.05 & 1 & $\ldots$ & 1.12 & 1 & $\ldots$ & 1.01 & 1 & $\ldots$ & $\ldots$ & $\ldots$ & $\ldots$ & $\ldots$ & $\ldots$ & \\
\hline $\mathrm{Zn} \mathrm{I}$ & 0.14 & 2.35 & 2 & 0.11 & 2.33 & 2 & 0.05 & 2.29 & 2 & 0.01 & 2.33 & 2 & 0.05 & 2.37 & 2 & 0.17 \\
\hline Sr II & 0.01 & 0.52 & 2 & 0.14 & 0.45 & 2 & 0.16 & 0.52 & 2 & 0.06 & 0.52 & 2 & 0.12 & 0.48 & 2 & 0.05 \\
\hline Y II & -0.10 & -0.25 & 6 & 0.08 & -0.41 & 6 & 0.02 & -0.50 & 6 & 0.15 & -0.26 & 6 & 0.15 & -0.32 & 5 & 0.12 \\
\hline Zr II & 0.33 & 0.54 & 3 & 0.13 & 0.49 & 3 & 0.15 & 0.44 & 3 & 0.16 & 0.66 & 3 & 0.18 & 0.47 & 3 & 0.08 \\
\hline Ba II & -0.10 & -0.35 & 4 & 0.08 & -0.44 & 4 & 0.07 & -0.41 & 4 & 0.07 & -0.44 & 4 & 0.12 & -0.45 & 4 & 0.06 \\
\hline La II & -0.15 & -1.40 & 4 & 0.22 & -1.26 & 5 & 0.08 & -1.37 & 5 & 0.05 & -1.07 & 4 & 0.13 & -1.20 & 4 & 0.13 \\
\hline Ce II & 0.11 & -0.73 & 4 & 0.23 & -0.92 & 5 & 0.16 & -1.04 & 4 & 0.11 & -0.71 & 1 & $\ldots$ & -0.45 & 1 & $\ldots$ \\
\hline $\mathrm{Nd}$ II & 0.25 & -0.64 & 7 & 0.14 & -0.81 & 10 & 0.10 & -0.77 & 10 & 0.08 & -0.63 & 4 & 0.18 & -0.83 & 2 & 0.09 \\
\hline Sm II & $\ldots$ & $\ldots$ & $\ldots$ & $\ldots$ & $\ldots$ & $\ldots$ & $\ldots$ & -1.30 & 2 & 0.02 & $\ldots$ & $\ldots$ & $\ldots$ & $\ldots$ & $\ldots$ & $\ldots$ \\
\hline Eu II & 0.40 & -1.48 & 2 & 0.04 & -1.72 & 4 & 0.20 & -1.45 & 3 & 0.05 & -1.38 & 2 & 0.04 & -1.51 & 2 & 0.01 \\
\hline Dy II & 0.64 & -0.65 & 1 & $\ldots$ & -0.68 & 2 & 0.27 & -0.98 & 2 & 0.30 & -0.71 & 1 & $\ldots$ & -1.13 & 1 & $\ldots$ \\
\hline
\end{tabular}

Notes.

a Star IDs from online version of Stetson (2005) or from Alcaino \& Liller (1980).

b Number of detected lines.

c Dispersion about the mean abundance.

d Synthesis of $\mathrm{CH}$ band near $4300 \AA$.

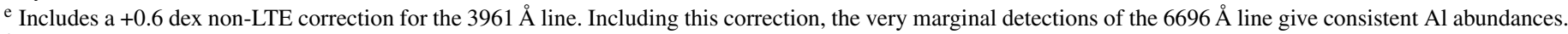

${ }^{\mathrm{f}}[\mathrm{Fe} / \mathrm{H}]$ from $\mathrm{Fe}$ I lines. All $[\mathrm{X} / \mathrm{Fe}]$ refer to $[\mathrm{Fe} / \mathrm{H}]$ from $\mathrm{Fe} \mathrm{I}$.

pattern is also observed in other distant clusters: NGC 7492 $\left(R_{G} \sim 25 \mathrm{kpc}\right.$; Cohen \& Melendez 2005b), the low luminosity GC Pal 3 ( $R_{G} \sim 90 \mathrm{kpc}$; Koch et al. 2009), and in co-additions of high-resolution, low-S/N spectra of 19 luminous giants in the very distant, low-luminosity GC Pal $4\left(R_{G} \sim 109 \mathrm{kpc}\right.$; Koch \& Côté 2010). The low [C/Fe] ratios seen among the NGC 2419 and M30 luminous giants are common among such stars in inner halo GCs (see, e.g., Briley et al. 2002), presumably due to depletion via deep mixing.

When comparing Galactic GC abundances to those of dSph satellites of the Galaxy, it has become apparent via extensive surveys in recent years (Cohen \& Huang 2009, 2010) and at moderate resolution the extensive work of Kirby et al. (2011) that the metal-poor end of all known systems seems to have, at least to first order, an identical chemical inventory. That should not be a surprise, since differences produced through different star formation rates or gas flow history (accretion and/or outflows) between the $\mathrm{dSph}$ satellites and the Galactic GCs will only show up at late times. Initially only the metals produced in and ejected by Type II supernova (SNII) enrich the system's gas, and hence, except for small metallicity dependent effects on nucleosynthetic yields, all very metal-poor stellar systems should have identical abundance ratios. Those GCs with peculiar abundance ratios, including no enhancement of the $\alpha$ elements, have intermediate $[\mathrm{Fe} / \mathrm{H}]$ and most are known to be associated with the ongoing accretion of the Sgr dSph; examples include Pal 12 (Cohen 2004) with $[\mathrm{Fe} / \mathrm{H}] \sim-0.7$ dex, Terzan 7 (Sbordone et al. 2005) with $[\mathrm{Fe} / \mathrm{H}]-0.6 \mathrm{dex}$, as well as Rup 106 (Brown et al. 1997) with $[\mathrm{Fe} / \mathrm{H}]-1.45$ dex, whose age is several Gyr less than the bulk of the halo, suggesting an accretion origin.

\section{COMPARISON WITH PREVIOUSLY PUBLISHED ABUNDANCE ANALYSES}

The only previously published high-dispersion analysis of any star in NGC 2419 is for the star S1305, which was observed by Shetrone et al. (2001). ${ }^{7}$ Our measurement of $[\mathrm{Fe} / \mathrm{H}]$ is $0.13 \mathrm{dex}$ higher than the value they obtained, which we ascribe largely to our adopted $T_{\text {eff }}$ being $125 \mathrm{~K}$ higher than theirs. The two sets of $[\mathrm{X} / \mathrm{Fe}]$ for all elements in common through atomic number $30(\mathrm{Zn})$ are in good agreement except for $\mathrm{Na} . \mathrm{Na}$ is

\footnotetext{
7 Shetrone et al. (2001) call this star RH 10. The coordinates of this star, privately communicated from M. Shetrone (2009), match those of S1305.
} 
Table 8

Abundance Sensitivities-Absolute Abundances $[\mathrm{X} / \mathrm{H}]$

\begin{tabular}{|c|c|c|c|c|}
\hline Species & $\begin{array}{c}T_{\text {eff }} \\
(+250 \mathrm{~K})^{\mathrm{a}} \\
(\mathrm{dex})\end{array}$ & $\begin{array}{c}\log (g) \\
(-0.5 \text { dex })^{b} \\
(\operatorname{dex})\end{array}$ & $\begin{array}{c}{[\mathrm{Fe} / \mathrm{H}](\text { model })} \\
(-0.5 \mathrm{dex}) \\
(\mathrm{dex})\end{array}$ & $\begin{array}{c}v_{t} \\
\left(+0.2 \mathrm{~km} \mathrm{~s}^{-1}\right) \\
(\operatorname{dex})\end{array}$ \\
\hline $\mathrm{C}(\mathrm{CH})^{\mathrm{c}}$ & 0.28 & 0.15 & -0.13 & -0.07 \\
\hline $\mathrm{OI}^{\mathrm{d}}$ & 0.04 & -0.21 & -0.13 & -0.01 \\
\hline $\mathrm{Na} I$ & 0.57 & 0.22 & 0.06 & -0.10 \\
\hline $\mathrm{Mg}_{\mathrm{I}}$ & 0.23 & 0.16 & 0.18 & -0.08 \\
\hline $\mathrm{Al} \mathrm{I}$ & 0.34 & 0.20 & 0.14 & -0.04 \\
\hline $\mathrm{K}_{\mathrm{I}}$ & 0.42 & 0.05 & 0.13 & -0.07 \\
\hline $\mathrm{Ca} \mathrm{I}$ & 0.28 & 0.09 & 0.12 & -0.04 \\
\hline Sc II & 0.00 & -0.16 & -0.08 & -0.03 \\
\hline Ti I & 0.63 & 0.08 & 0.21 & -0.05 \\
\hline Ti II & -0.03 & -0.04 & 0.01 & -0.10 \\
\hline V I & 0.54 & 0.06 & 0.14 & 0.00 \\
\hline Cr I & 0.55 & 0.12 & 0.23 & -0.08 \\
\hline Mn I & 0.42 & 0.11 & 0.17 & -0.04 \\
\hline $\mathrm{Fe} I$ & 0.42 & 0.06 & 0.13 & -0.07 \\
\hline Fe II & -0.17 & -0.08 & 0.00 & -0.09 \\
\hline Co I & 0.45 & 0.05 & 0.11 & -0.01 \\
\hline $\mathrm{Ni} I$ & 0.42 & 0.02 & 0.09 & -0.02 \\
\hline $\mathrm{Cu}$ & 0.44 & 0.06 & 0.12 & -0.01 \\
\hline Sr I & 0.44 & 0.12 & 0.17 & 0.00 \\
\hline Sr II & 0.10 & 0.04 & -0.02 & -0.07 \\
\hline Y II & 0.02 & -0.11 & -0.03 & -0.03 \\
\hline Ba II & 0.13 & -0.16 & -0.08 & -0.14 \\
\hline La II & 0.09 & -0.14 & -0.06 & -0.02 \\
\hline Ce II & 0.07 & -0.10 & -0.03 & -0.02 \\
\hline Nd II & 0.10 & -0.14 & -0.07 & -0.02 \\
\hline Eu II & 0.06 & -0.08 & -0.02 & -0.09 \\
\hline
\end{tabular}

\section{Notes.}

${ }^{a} T_{\text {eff }}$ changes from 4250 to $4500 \mathrm{~K}$.

${ }^{\mathrm{b}} \log (g)$ changes from 1.0 to 0.5 dex.

c The $4320 \AA$ region of the $G$ band of $\mathrm{CH}$ was used.

d Values for the [O I] lines 6300, $6363 \AA$.

a difficult case as the $\mathrm{NaD}$ lines are somewhat corrupted by interstellar features at the cluster's $v_{r}$, while the $5682,5688 \AA$ doublet used by Shetrone et al. (2001) is very weak. Among the heavier elements detected in both studies, only Y (in the form of Y II) has a large discrepancy. There are three detected lines from our spectra, and a claim of four from theirs, but the line at $4900.1 \AA$ is quite crowded, and perhaps should be ignored. For the two Y II lines in common, our $W_{\lambda}$ are $\sim 20 \%$ smaller. Considering how faint this star is $(V=17.61 \mathrm{mag})$ we regard the overall agreement in abundances between the two analyses to be very good.

Shetrone et al. (2003) observed one star in NGC 7099. Once the difference in adopted solar $\mathrm{Fe}$ abundance is taken into account, their derived $[\mathrm{Fe} / \mathrm{H}]$ for this star differs from the mean for our sample of five stars by only 0.05 dex. The abundance ratios agree satisfactorily in most cases, with seven elements differing in $[\mathrm{X} / \mathrm{Fe}]$ by less than 0.10 dex. Their UVES observations cover only $4800-6800 \AA$, and so are missing many key blue lines for the rare earths, where the differences in relative abundances between the two studies tend to be the largest. The choice of adopted transition probabilities, particularly for the rare earths, also contributes; we adopted them from the recent studies of Lawler and collaborators (see, e.g., Lawler et al. 2001, for Eu as an example) when available, while Shetrone et al. (2003) tended to use older values.

The Padua group (see, e.g., Carretta et al. 2009a, 2009b, 2009c, 2010) has completed a large VLT study of the $\mathrm{Na} / \mathrm{O}$

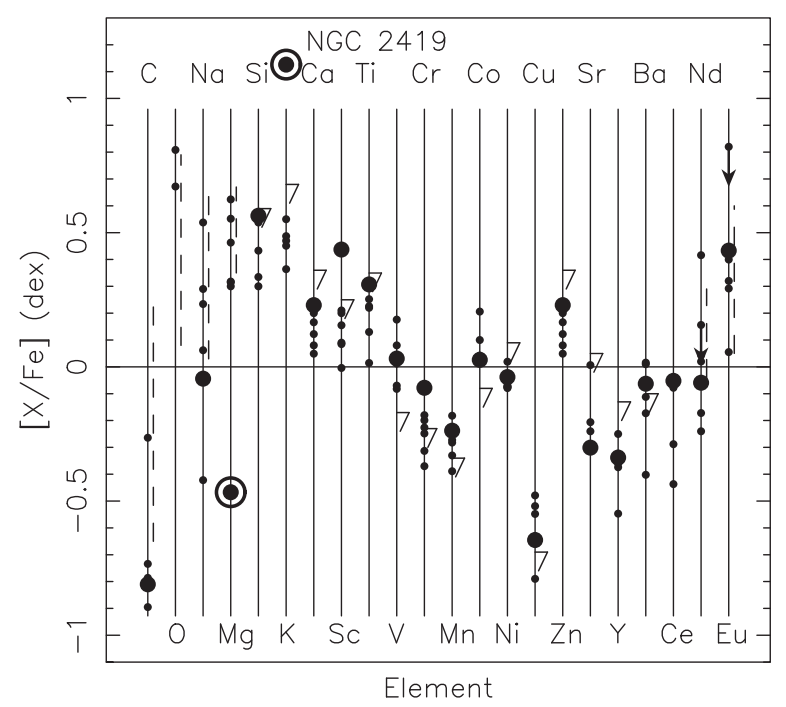

Figure 4. Abundance ratios for the detected elements in the NGC 2419 sample of seven red giants, with the large filled circles indicating S1131. The highly unusual abundance ratios of $[\mathrm{Mg} / \mathrm{Fe}]$ and $[\mathrm{K} / \mathrm{Fe}]$ for NGC $2419 \mathrm{~S} 1131$ are circled. The reference species is Fe I for neutral species and Fe II for ionized ones. The averages of Ti I and Ti II and of Sr I and Sr II are plotted when both are available. Average abundance ratios from our analyses of HIRESr spectra of red giants in the inner halo cluster M30 (NGC 7099) are indicated by "7." For elements with a large range in $\mathrm{M} 30$, the range is indicated by a dashed line.

anti-correlation in GCs. Although their survey does not include NGC 2419, they have observed a sample of 10 RGB stars in M30 with the Ultraviolet and Visual Echelle Spectrograph (UVES). Thus far they have published abundances for only the light elements. Table 11 presents a comparison of our results from HIRES at Keck with their UVES/VLT study and highlights the good agreement between these two completely independent detailed abundance analyses for RGB stars in M30.

\section{CONSISTENCY WITH OUR PREVIOUS DEIMOS RANGE IN Ca ABUNDANCE}

Our previous medium-resolution study of RGB stars in NGC 2419 (Cohen et al. 2010) uncovered a range in $[\mathrm{Ca} / \mathrm{H}]$ of a factor of two or three among the sample of 43 stars found to be definite members of this GC. The range in $V$ of these stars is from 17.4 to $19.3 \mathrm{mag}$. Figure 5 shows a histogram of $[\mathrm{Ca} / \mathrm{H}]$ for these stars, on which is superposed the distribution of the giants in NGC 2419 with high quality HIRES spectra analyzed here. The two additional probable members discussed in Cohen et al. (2010), each of which has some remaining concern about membership, are indicated in this figure with cross hatching.

The first point to note is that the histogram of $[\mathrm{Ca} / \mathrm{H}]$ for an unbiased sample of members of NGC 2419, the moderate resolution Deimos survey of Cohen et al. (2010), is sharply peaked at $[\mathrm{Ca} / \mathrm{H}]-1.95 \mathrm{dex}$. While there is a rapidly declining tail extending toward higher metallicities, the low metallicity peak dominates, and the fraction of stars in the extended tail is small. The minimum $[\mathrm{Ca} / \mathrm{H}]$ is -2.0 dex. Only $13(30 \%)$ of the 43 definite members lie outside the range -2.0 to -1.85 dex.

Our sample of RGB stars in NGC 2419 with HIRES spectra good enough for a detailed abundance analysis contains only seven stars. This sample was, to a large extent, selected by brightness before the full analysis of the moderate resolution spectra was completed. One of these seven stars (S1131) has $[\mathrm{Ca} / \mathrm{H}]-1.72$ dex from its HIRES spectrum $(-1.76$ dex from its Deimos Ca triplet region analysis) and a second star (S973) is marginally above the $-1.85 \mathrm{dex}[\mathrm{Ca} / \mathrm{H}]$ cutoff at $-1.81 \mathrm{dex}$ 
Table 9

Abundance Ratio Sensitivities

\begin{tabular}{|c|c|c|c|c|c|c|}
\hline Species & $\begin{array}{c}\text { rms Total }^{\mathrm{a}} \\
\text { (dex) }\end{array}$ & $\begin{array}{c}\Delta T_{\text {eff }} \\
(100 \mathrm{~K}) \\
(\operatorname{dex})\end{array}$ & $\begin{array}{c}\Delta \log (g) \\
(0.1 \text { dex })^{\mathrm{b}} \\
\quad(\operatorname{dex})\end{array}$ & $\begin{array}{c}\Delta[\mathrm{Fe} / \mathrm{H}](\text { model }) \\
(0.1 \operatorname{dex}) \\
(\operatorname{dex})\end{array}$ & $\begin{array}{c}\Delta v_{t} \\
\left(+0.2 \mathrm{~km} \mathrm{~s}^{-1}\right) \\
(\operatorname{dex})\end{array}$ & $\begin{array}{l}W_{\lambda}{ }^{\mathrm{b}} \\
(\mathrm{dex})\end{array}$ \\
\hline $\mathrm{C}(\mathrm{CH})^{\mathrm{c}}$ & 0.11 & -0.06 & 0.02 & -0.05 & 0.00 & 0.08 \\
\hline$\left[\mathrm{O}_{\mathrm{I}} / \mathrm{Fe}_{\mathrm{I}}\right]^{\mathrm{d}}$ & 0.13 & 0.08 & -0.03 & -0.05 & -0.01 & 0.08 \\
\hline$[\mathrm{Na} \mathrm{I} / \mathrm{Fe} \mathrm{I}]$ & 0.15 & 0.06 & 0.03 & -0.03 & -0.10 & 0.08 \\
\hline$[\mathrm{Mg} \mathrm{I} / \mathrm{Fe} \mathrm{I}]$ & 0.12 & -0.08 & 0.02 & 0.02 & -0.08 & 0.05 \\
\hline$[\mathrm{Al} \mathrm{I} / \mathrm{Fe} \mathrm{I}]$ & 0.08 & -0.03 & 0.03 & 0.00 & -0.04 & 0.06 \\
\hline$\left[\mathrm{K}_{\mathrm{I}} / \mathrm{Fe}_{\mathrm{I}}\right]$ & 0.11 & 0.00 & 0.00 & 0.00 & -0.07 & 0.08 \\
\hline$[\mathrm{Ca} \mathrm{I} / \mathrm{Fe} \mathrm{I}]$ & 0.07 & -0.06 & 0.01 & 0.00 & -0.04 & 0.02 \\
\hline$[\mathrm{Sc}$ II $/ \mathrm{Fe}$ II $]$ & 0.09 & 0.07 & -0.02 & -0.03 & -0.03 & 0.03 \\
\hline$[\mathrm{Ti} \mathrm{I} / \mathrm{Fe} \mathrm{I}]$ & 0.11 & 0.08 & 0.00 & 0.03 & -0.05 & 0.02 \\
\hline [Ti II/Fe II] & 0.12 & 0.06 & 0.01 & 0.00 & -0.10 & 0.03 \\
\hline$\left[\mathrm{V} \mathrm{I}_{\mathrm{I}} / \mathrm{Fe} \mathrm{I}_{\mathrm{I}}\right]$ & 0.07 & 0.05 & 0.00 & 0.00 & 0.00 & 0.05 \\
\hline$[\mathrm{Cr} \mathrm{I} / \mathrm{Fe} \mathrm{I}]$ & 0.11 & 0.05 & 0.01 & 0.04 & -0.08 & 0.03 \\
\hline$\left[\mathrm{Mn} \mathrm{I} / \mathrm{Fe} \mathrm{I}_{\mathrm{I}}\right]$ & 0.06 & 0.00 & 0.01 & 0.02 & -0.04 & 0.03 \\
\hline$\left[\mathrm{Fe}_{\mathrm{I}} / \mathrm{Fe}_{\mathrm{I}}\right]$ & 0.07 & 0.00 & 0.00 & 0.00 & -0.07 & 0.01 \\
\hline$\left[\mathrm{Fe}_{\mathrm{II}} / \mathrm{Fe}_{\mathrm{I}}\right]$ & 0.26 & -0.24 & -0.03 & -0.05 & -0.09 & 0.03 \\
\hline$[\mathrm{Fe}$ II $/ \mathrm{Fe} \mathrm{II}]$ & 0.09 & 0.00 & 0.00 & 0.00 & -0.09 & 0.03 \\
\hline$\left[\mathrm{CoI} / \mathrm{Fe}_{\mathrm{I}}\right]$ & 0.08 & 0.01 & 0.00 & -0.01 & -0.01 & 0.08 \\
\hline [Ni I/Fe I] & 0.04 & 0.00 & -0.01 & -0.02 & -0.02 & 0.02 \\
\hline$[\mathrm{Cu}$ I/Fe I $]$ & 0.08 & 0.01 & 0.00 & 0.00 & -0.01 & 0.08 \\
\hline$[\mathrm{Sr} \mathrm{I} / \mathrm{Fe} \mathrm{I}]$ & 0.08 & 0.01 & 0.01 & 0.02 & 0.00 & 0.08 \\
\hline [Sr II/Fe II] & 0.15 & 0.11 & 0.02 & -0.01 & -0.07 & 0.08 \\
\hline [Y II/Fe II] & 0.09 & 0.08 & -0.01 & -0.01 & -0.03 & 0.04 \\
\hline$[\mathrm{Ba} \mathrm{II} / \mathrm{Fe} \mathrm{II}]$ & 0.19 & 0.12 & -0.02 & -0.03 & -0.14 & 0.05 \\
\hline [La II/Fe II] & 0.14 & 0.10 & -0.01 & -0.02 & -0.02 & 0.08 \\
\hline$[\mathrm{Ce}$ II $/ \mathrm{Fe}$ II $]$ & 0.11 & 0.10 & 0.00 & -0.01 & -0.02 & 0.06 \\
\hline$[\mathrm{Nd}$ II $/ \mathrm{Fe} \mathrm{II}]$ & 0.13 & 0.11 & -0.01 & -0.03 & -0.02 & 0.06 \\
\hline$[\mathrm{Eu} \mathrm{II} / \mathrm{Fe} \mathrm{II}]$ & 0.14 & 0.09 & 0.00 & -0.01 & -0.09 & 0.06 \\
\hline
\end{tabular}

Notes.

a The rms sum of all five contributing terms, relative to whichever of Fe I or Fe II gave the smaller value.

b Assumed to be $0.08 \mathrm{dex} / \sqrt{N(\text { lines })}$.

c The $4320 \AA$ region of the $G$ band of $\mathrm{CH}$ was used.

d Values for the [O I] lines 6300, $6363 \AA$.

from our detailed abundance analysis $(-1.93$ dex from its moderate resolution Deimos spectrum). The others all are more metal-poor than $[\mathrm{Ca} / \mathrm{H}]=-1.85 \mathrm{dex}$ as deduced from both their HIRES and Deimos spectra.

The total range in $\mathrm{Ca}$ and $\mathrm{Fe}$ abundance within the NGC 2419 sample is small (see Table 6). We used the three different choices of $T_{\text {eff }}$ discussed in Section 2 and given in Table 2 as an indication of the maximum range in this parameter that might be appropriate for each of the NGC 2419 stars. ${ }^{8}$ In effect, we carried out the abundance analysis three times for each star using different sets of adopted stellar parameters. We find that for each of these three choices, S1131 always has the highest value of $[\mathrm{Ca} / \mathrm{H}]$ of the seven stars in the HIRES sample for NGC 2419 , and it is $\sim 0.2$ dex ( $2 \sigma$ to $3 \sigma$ ) higher than the mean value for the other six stars in this cluster. The Fe abundance for S1131, as derived from either Fe I or Fe II, is always the highest or second highest as well, but is within $1 \sigma$ of the mean of the other cluster stars.

The uncertainties in absolute abundances (see Table 8) are daunting for such a small range in $\mathrm{Ca}$ abundance in such a distant cluster with spectra and near-IR photometry that are less than ideal. Lowering the $T_{\text {eff }}$ of S1131 in NGC 2419 by $\sim 100 \mathrm{~K}$ relative to the other RGB stars in this cluster in our HIRES sample would eliminate its high $\mathrm{Ca}$ and $\mathrm{Fe}$ abundances. But

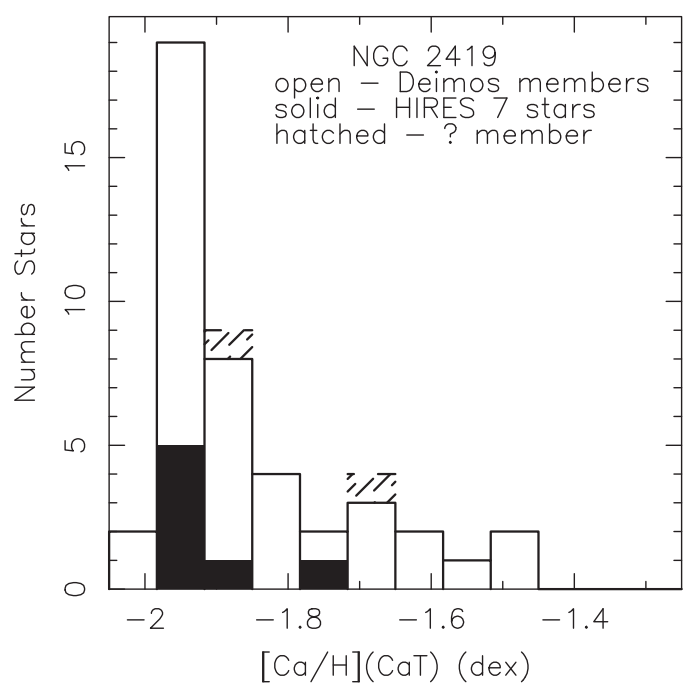

Figure 5. Histogram of $[\mathrm{Ca} / \mathrm{H}]$ as inferred from the Deimos moderate resolution spectra of Cohen et al. (2010) is shown for the sample of 43 definite members of NGC 2419 isolated in that paper. The present sample of seven RGB stars in this GC with HIRES spectra is shown by the solid fill. The two additional stars in the Deimos survey which are probable members, both of which have low $\mathrm{S} / \mathrm{N}$ HIRES spectra, are indicated by the hatched areas, which are placed above the histogram defined by the definite members.

\footnotetext{
8 We adjusted $\log (g)$ appropriately in each case to maintain the star on the RGB.
} 
Table 10

Mean Abundances for Red Giant Members of NGC 2419 and of NGC 7099

\begin{tabular}{|c|c|c|c|c|c|c|}
\hline Species & $\begin{array}{c}\text { NGC } \\
\langle[\mathrm{X} / \mathrm{Fe}]\rangle^{\mathrm{a}} \\
\quad(\operatorname{dex})\end{array}$ & $\begin{array}{c}7099 \\
\sigma[\mathrm{X} / \mathrm{Fe}] \\
(\mathrm{dex})\end{array}$ & No. of Stars & $\begin{array}{c}\text { NGC } \\
\langle[\mathrm{X} / \mathrm{Fe}]\rangle^{\mathrm{a}} \\
(\mathrm{dex})\end{array}$ & $\begin{array}{c}2419 \\
\sigma[\mathrm{X} / \mathrm{Fe}] \\
(\mathrm{dex})\end{array}$ & No. of Stars \\
\hline $\log [\epsilon(\mathrm{Fe})](\mathrm{Fe} \mathrm{I})$ & -2.42 & 0.07 & 5 & -2.06 & 0.10 & 7 \\
\hline $\log [\epsilon(\mathrm{Fe})](\mathrm{Fe}$ II $)$ & -2.38 & 0.04 & 5 & -2.12 & 0.07 & 7 \\
\hline$[\mathrm{C} / \mathrm{Fe}]$ & -0.28 & 0.35 & 5 & -0.70 & 0.25 & 5 \\
\hline$[\mathrm{O} / \mathrm{Fe}]$ & 0.35 & 0.28 & 5 & 0.74 & 0.10 & 2 \\
\hline$[\mathrm{Na} / \mathrm{Fe}]$ & 0.29 & 0.27 & 5 & 0.09 & 0.31 & 7 \\
\hline$[\mathrm{Mg} / \mathrm{Fe}]$ & 0.51 & 0.11 & 5 & 0.30 & 0.36 & 7 \\
\hline$[\mathrm{Al} / \mathrm{Fe}]^{\mathrm{b}}$ & 0.51 & 0.36 & 5 & 0.45 & $\ldots$ & 1 \\
\hline$[\mathrm{Si} / \mathrm{Fe}]$ & 0.52 & 0.07 & 5 & 0.43 & 0.12 & 5 \\
\hline$[\mathrm{K} / \mathrm{Fe}]$ & 0.61 & 0.10 & 5 & 0.58 & 0.28 & 6 \\
\hline$[\mathrm{Ca} / \mathrm{Fe}]$ & 0.29 & 0.05 & 5 & 0.15 & 0.07 & 7 \\
\hline$[\mathrm{Sc}$ II $/ \mathrm{Fe} \mathrm{II}]$ & 0.18 & 0.03 & 5 & 0.17 & 0.14 & 7 \\
\hline$[\mathrm{Ti} / \mathrm{Fe}]$ & 0.21 & 0.05 & 5 & 0.11 & 0.07 & 7 \\
\hline [Ti II/Fe II] & 0.35 & 0.03 & 5 & 0.30 & 0.16 & 7 \\
\hline$[\mathrm{V} / \mathrm{Fe}]$ & -0.23 & 0.09 & 5 & 0.03 & 0.09 & 7 \\
\hline$[\mathrm{Cr} / \mathrm{Fe}]$ & -0.30 & 0.03 & 5 & -0.23 & 0.09 & 7 \\
\hline$[\mathrm{Mn} / \mathrm{Fe}]$ & -0.40 & 0.06 & 5 & -0.28 & 0.07 & 7 \\
\hline$[\mathrm{Fe} \mathrm{II} / \mathrm{Fe} \mathrm{I}]$ & 0.05 & 0.05 & 5 & -0.06 & 0.05 & 7 \\
\hline$[\mathrm{Co} / \mathrm{Fe}]$ & -0.16 & 0.12 & 5 & 0.09 & 0.08 & 4 \\
\hline$[\mathrm{Ni} / \mathrm{Fe}]$ & 0.02 & 0.07 & 5 & -0.05 & 0.03 & 7 \\
\hline$[\mathrm{Cu} / \mathrm{Fe}]$ & -0.76 & 0.17 & 3 & -0.61 & 0.10 & 7 \\
\hline$[\mathrm{Zn} / \mathrm{Fe}]$ & 0.16 & 0.07 & 5 & -0.20 & 0.06 & 4 \\
\hline$[\mathrm{Sr} / \mathrm{Fe}]$ & $\ldots$ & $\ldots$ & $\ldots$ & -0.23 & 0.13 & 4 \\
\hline [Sr II/Fe II] & -0.03 & 0.05 & 5 & -0.08 & 0.12 & 3 \\
\hline [Y II/Fe II] & -0.23 & 0.15 & 5 & -0.36 & 0.09 & 7 \\
\hline [Ba II/Fe II] & -0.18 & 0.04 & 5 & -0.11 & 0.14 & 7 \\
\hline [La II/Fe II] & -0.02 & 0.17 & 5 & 0.05 & 0.30 & 4 \\
\hline$[\mathrm{Nd}$ II $/ \mathrm{Fe} \mathrm{II}]$ & 0.14 & 0.10 & 5 & 0.00 & 0.23 & 6 \\
\hline [Eu II/Fe II] & 0.41 & 0.18 & 5 & 0.30 & 0.15 & 5 \\
\hline
\end{tabular}

Notes.

${ }^{\mathrm{a}}[\mathrm{X} / \mathrm{Fe}]$ where both species are neutral, unless otherwise indicated.

b A non-LTE correction of +0.6 dex has been applied to abundances derived from the $3961 \AA$ line of Al I.

this requires a change of this one star relative to all the others which is about half of the entire range in $T_{\text {eff }}$ spanned by the seven cluster giants, for which there is no justification. Given the small size of our HIRES sample, all we can say is that the $\mathrm{Ca} / \mathrm{H}$ distribution of the HIRES sample in NGC 2419 is consistent with that of the Deimos sample, which does appear to show a small but measurable range in $\mathrm{Ca} / \mathrm{H}$ among the cluster members (Cohen et al. 2010).

Both of two probable additional members of NGC 2419 discussed in Cohen et al. (2010) from the Deimos sample, shown by cross hatching in Figure 5, have been observed with HIRES. However observations were terminated after the first 30 minute exposure revealed concerns about cluster membership. Hence the resulting HIRES spectra for these two stars are not good enough for a detailed abundance analysis. One of these (S951) has a spectrum which appears to be that of an RGB star in this very metal-poor GC, but with $v_{r}$ off from the cluster mean by $\sim 15 \mathrm{~km} \mathrm{~s}^{-1}$. This star has a value from an analysis of the $\mathrm{Ca}$ triplet lines in its Deimos spectrum of $[\mathrm{Ca} / \mathrm{H}]$ of $-1.91 \mathrm{dex}$, consistent with the bulk of the cluster population. The concern with the second star, S1673, is twofold; it lies slightly bluer than the main RGB locus of NGC 2419 in $V-I$ (see Figure 2) and its HIRES spectrum appears metal-rich. The $[\mathrm{Ca} / \mathrm{H}]$ inferred from its Deimos spectrum $(-1.68 \mathrm{dex})$ puts this star, if it is a member, firmly in the metal-rich tail of the histogram shown in
Table 11

Comparison of Our Mean Abundances for Red Giant Members of NGC 7099 with Those from the Carretta et al. VLT Large Program

\begin{tabular}{lccc}
\hline \hline Species & $\begin{array}{c}\text { Carretta et al. } \begin{array}{c}\text { a,b } \\
(\text { dex }) \\
(\text { Mean, } \sigma)\end{array} \\
{[\mathrm{Fe} / \mathrm{H}]}\end{array}$ & $\begin{array}{c}\text { This Paper }^{\mathrm{c}} \\
(\text { dex }) \\
(\text { Mean, } \sigma)\end{array}$ & Notes \\
{$[\mathrm{O} / \mathrm{Fe}]$} & $-2.35(0.05)$ & $-2.42(0.07)$ & \\
{$[\mathrm{Na} / \mathrm{Fe}]$} & $0.46(0.20)$ & $0.35(0.28)$ & $\mathrm{d}, \mathrm{e}$ \\
{$[\mathrm{Mg} / \mathrm{Fe}]$} & $0.35(0.25)$ & $0.29(0.27)$ & $\mathrm{d}$ \\
{$[\mathrm{Al} / \mathrm{Fe}]$} & $0.51(0.04)$ & $0.51(0.11)$ & $\mathrm{d}, \mathrm{e}$ \\
{$[\mathrm{Si} / \mathrm{Fe}]$} & $0.77(0.32)$ & $0.51(0.36)$ & \\
{$[\mathrm{Ca} / \mathrm{Fe}]$} & $0.34(0.07)$ & $0.52(0.07)$ & $\mathrm{f}, \mathrm{g}$ \\
{$[\mathrm{Ba} / \mathrm{Fe}]$} & $0.31(0.03)$ & $0.29(0.05)$ & $-0.22(0.14)$ \\
\hline
\end{tabular}

Notes.

a Carretta et al. (2009a, 2009b, 2009c, 2010).

${ }^{\mathrm{b}}$ UVES sample of 10 RGB stars in NGC 7099.

c Our sample of five RGB stars.

$\mathrm{d}$ This element has a large internal abundance range.

e There are many upper limits in the VLT sample, which E. Carretta (2011, private communication) advises they treated as detections to calculate their mean abundance ratios.

${ }^{f}$ From the large intermediate resolution FLAMES/VLT survey; see D'Orazi et al. (2010).

$\mathrm{g}$ This, for our result, is the ratio [Ba II/Fe II]. 
Figure 5. See Cohen et al. (2010) for further discussion regarding membership in NGC 2419 of these two stars.

There are 13 stars in the Deimos sample that may be members of NGC 2419 with $V<17.7$, which is slightly fainter than the faintest star in the present HIRES sample analyzed here. In addition to all seven RGB stars in our present HIRES sample, both of the two probable cluster members discussed above are brighter than that cutoff. This leaves four stars brighter than $V=17.7$ mag without HIRES observations at present, three of which have $[\mathrm{Ca} / \mathrm{H}] \sim-1.9$ dex. Only one is in the high metallicity tail for NGC 2419.

Our present HIRES sample, which was selected primarily on the basis of brightness given the $84 \mathrm{kpc}$ distance to NGC 2419, is not ideal for exploring the star-to-star variation. The small variations of $[\mathrm{Ca} / \mathrm{H}]$ or $[\mathrm{Fe} / \mathrm{H}]$ from the seven stars with high dispersion spectra are only marginally larger than the uncertainties, but are broadly consistent with our Deimos results (Cohen et al. 2010) which found a range in $[\mathrm{Ca} / \mathrm{H}]$ within NGC 2419. In the future we hope to provide a sample that better probes the metal-rich tail of NGC 2419 as defined by the larger Deimos sample of Cohen et al. (2010).

\section{ONE STAR WITH A PECULIAR ABUNDANCE PATTERN}

One star, NGC 2419 S1131, deviates from the archetypal GC abundance pattern. S1131 is a definite member of NGC 2419 based on its HIRES radial velocity (its heliocentric $v_{r}$ is $-17.2 \mathrm{~km} \mathrm{~s}^{-1}$ ) and spectrum; see also Cohen et al. (2010). As discussed above in Section 6 our detailed abundance analysis suggests that this star is slightly more metal-rich than the other NGC 2419 giants in Fe and $\mathrm{Ca}$ as well as several other elements with many detected lines. It has an enhancement of $\sim 0.2 \mathrm{dex}$ in $[\mathrm{Ca} / \mathrm{H}]$ (with slight variation depending on the choice of $T_{\text {eff }}$ discussed in Section 3), corresponding to $\mathrm{Ca}$ enhanced by a factor of 1.6, compared to the mean of the other six NGC 2419 giants.

It is interesting to note that S1131 is the only one of the six NGC 2419 sample giants included in the moderate resolution study of Cohen et al. (2010) found to be Ca-rich. The remaining six NGC 2419 giants in the HIRES sample all lie at the low $\mathrm{Ca}$ abundance end of the distribution inferred by Cohen et al. (2010). However, given the small abundance range involved, observations of additional giants believed to be more metalrich than the bulk of the cluster stars are required for a definitive confirmation for star-to-star variation of $\mathrm{Ca}, \mathrm{Fe}$, and other heavy elements within NGC 2419.

Furthermore, NGC 2419 S1131 has some anomalous abundance ratios, irrespective of whether its $T_{\text {eff }}$ has been overestimated. It has $[\mathrm{Mg} / \mathrm{Fe}]=-0.47$ dex from five $\mathrm{Mg}$ I lines, in contrast to the mean of the other six stars, $+0.44 \pm 0.06$ dex. In deriving the $\mathrm{Mg}$ abundance for $\mathrm{S} 1131$, the upper limit to the strength of the $5711 \AA$ line was considered as a detection, and the two unblended $\mathrm{Mg}$ triplet lines were retained. These two lines give $\mathrm{Mg}$ abundances slightly higher than the mean, but the highest $\mathrm{Mg}$ abundance is from the $5711 \AA$ line, $0.18 \mathrm{dex}$ above the mean of the five lines used. Note that the first ionization potential of $\mathrm{Mg}$ is only $0.25 \mathrm{eV}$ lower than that of Fe. The $[\mathrm{Mg} / \mathrm{Fe}]$ measurement would increase by only 0.08 dex if $T_{\text {eff }}$ were reduced by $100 \mathrm{~K}$ (see Table 9 ). The regions of the spectra of two Mg I lines in this star and in S1209, also known as Suntzeff 16 (Suntzeff et al. 1988), with $T_{\text {eff }}$ lower than that of $\mathrm{S} 1131$ by only $85 \mathrm{~K}$, are shown in Figure 3. Although most GCs show a small spread in $[\mathrm{Mg} / \mathrm{Fe}]$ (Gratton et al. 2004), gener- ally interpreted as the result of proton-burning of $\mathrm{Mg}$ into $\mathrm{Al}$ in intermediate mass asymptotic giant branch stars, the expected enhancement of $\mathrm{Al}$ does not appear to be present in S1131. The abundance pattern of this specific star cannot be reproduced by proton-burning. Furthermore $-0.47 \mathrm{dex}$ is an unprecedentedly low $[\mathrm{Mg} / \mathrm{Fe}]$ abundance for a GC star; the only stars that have such low $[\mathrm{Mg} / \mathrm{Fe}]$ ratios are the halo star CS22966-043 (Ivans et al. 2003), a hot SX Phe variable, and stars in dSph satellite galaxies, such as COS 171 in Ursa Minor (Cohen \& Huang 2010) and S58 in Sextans (Shetrone et al. 2001). NGC 2419 S1131 is unique in that no other elements appear depleted, including Si. A very low value of $\mathrm{Mg}$ and super-solar values of other alpha elements are inconsistent with any Type II SN yields (e.g., Woosley \& Weaver 1995; Nomoto et al. 2006; Heger \& Woosley 2010).

In addition, NGC 2419 S1131 also has an unusually high $[\mathrm{K} / \mathrm{Fe}]$ of $+1.13 \mathrm{dex}$, also illustrated in Figure 3. This figure includes the spectrum of a rapidly rotating B star to demonstrate that any telluric absorption at the wavelength of the $\mathrm{K}_{\mathrm{I}}$ line, taking into account the $v_{r}$ of this GC, is negligible. Furthermore, two other NGC 2419 sample stars were observed on the same run (see Table 1) and do not show this abnormality. This abundance ratio would decrease by less than $0.10 \mathrm{dex}$ if $T_{\text {eff }}$ were reduced by $200 \mathrm{~K}$. This is an internal comparison within our NGC 2419 sample, which has a total range in $T_{\text {eff }}$ of less than $200 \mathrm{~K}$. Studies of non-LTE corrections for the $7699 \AA$ resonance line of K I (the only line used here) by Andrievsky et al. (2010) and by Takeda et al. (2010) suggest that the appropriate non-LTE corrections change by only 0.15 dex over such a small range in $T_{\text {eff }}$, ranging from about -0.35 to -0.2 dex. Thus, differential nonLTE corrections for $\mathrm{K}_{\mathrm{I}}$ within our HIRES sample of luminous RGB stars are much too small to explain the deviant potassium abundance of S1131.

Takeda et al. (2010) very recently found a red giant in M13 and another in M4 with similarly high $[\mathrm{K} / \mathrm{Fe}]$. They ascribe the unexpectedly strong $\mathrm{K}_{\mathrm{I}}$ resonance lines to an increase in activity or turbulent velocity fields high in the stellar atmosphere where the core of such a strong line is formed. However, while S1131 does show emission on both the red and blue wings of $\mathrm{H} \alpha$, the emission wings of $\mathrm{H} \alpha$ in the spectrum of NGC 2419 S223 are considerably stronger, yet this star does not show an anomalously strong $\mathrm{K}_{\mathrm{I}}$ line. Furthermore if this explanation is correct, other strong resonance lines arising from neutral species of elements with (low) first ionization potentials comparable to that of $\mathrm{K}$ should be similarly affected. Unfortunately the $\mathrm{NaD}$ lines, the most likely candidates to check, are badly corrupted by interstellar absorption due to the radial velocity of NGC 2419.

A careful inspection of Figure 3 strongly suggests, by comparison with the spectrum of NGC 2419 S1209, which has a $V$ magnitude only 0.2 mag brighter than S1131, that S1131 is indeed slightly more metal-rich in other species as well as $\mathrm{K}$.

The Li I blend at $6707 \AA$ was too weak to be detected in any of the NGC 2419 stars. The upper limit for the equivalent width of this feature of $7 \mathrm{m \AA}$ in S1131 corresponds to $\log [\epsilon(\mathrm{Li})]$ of $-0.01 \mathrm{dex}$, indicates extensive depletion from the Li production in the big bang, typical of red giants, which have extensive surface convection zones.

The stellar parameters, spectra, and analyses have been carefully checked and these specific differences in abundance ratios discussed here (i.e., for $\mathrm{Mg}$ and for $\mathrm{K}$ ) within the NGC 2419 sample are real. 


\section{SUMMARY}

Our key result from the analysis of seven luminous giant members of the distant outer halo globular cluster NGC 2419 is that one of these stars is extremely peculiar, having a very low $[\mathrm{Mg} / \mathrm{Fe}]$ ratio, but being normal in all other element ratios except for a high $[\mathrm{K} / \mathrm{Fe}]$. Similarly low $[\mathrm{Mg} / \mathrm{Fe}]$ ratios are seen in stars in dSph satellites of the Galaxy, specifically in Ursa Minor (Cohen \& Huang 2010) and in Sextans (Shetrone et al. 2001). But in both cases these stars have low values of other $\alpha$ elements, which is not the case for NGC 2419 S1131. J. Cohen has examined and analyzed spectra of $\gtrsim 100$ stars in Galactic GCs and has never seen one similar to S1131 in NGC 2419.

Furthermore, the HIRES spectra support the small range in metallicity within the giants in NGC 2419 found by Cohen et al. (2010), which might suggest that this distant outer halo GC is the remnant of an accreted dwarf galaxy. A definitive result will require further observations of the faint upper RGB stars in this $\mathrm{GC}$, concentrating on those whose moderate resolution Deimos spectra from Cohen et al. (2010) suggest that they are more metal-rich than the bulk of the stars in this cluster, which will be carried out in a future campaign.

Ignoring the limited peculiarities of S1131, we find that there is no substantive difference between the mean behavior of the abundance ratios for six other red giants in NGC 2419 and those ratios characteristic of the inner halo despite the structural peculiarities of NGC 2419. The influence of star formation rates and other aspects of the detailed chemical evolution of a stellar system only affect such ratios once star formation has been underway long enough for sources other than SNII to become important contributors, which does not seem to be the case in very metal-poor systems such as NGC 2419 nor in the most metal-poor stars in the dSph satellites of the Milky Way (Cohen \& Huang 2009, 2010; Kirby et al. 2011).

Thus, like the previous studies based on detailed abundance analyses of individual or co-added stellar spectra of stars in distant outer halo clusters, the chemical inventory of the GC system appears to be independent of location. With the exception of those clusters known to be associated with the currently disrupting Sgr dSph galaxy, every GC exhibits the same trends as a function of overall cluster metallicity - parameterized by $[\mathrm{Fe} / \mathrm{H}]$-irrespective of kinematics or location within the halo.

The existence of a metallicity gradient in the Galactic halo is controversial. Carollo et al. (2007) found evidence for a break in the metallicity distribution of halo field stars in the sense that the more distant stars are more metal-poor; this conclusion is supported by de Yong et al. (2010). Furthermore, Carollo et al. (2007) showed that the metallicity within the outer halo population displays a gradient toward even lower metallicities for the most distant stars or stars showing the highest magnitude of rotation retrograde to the Milky Way disk. However, both Sesar et al. (2011) and Schoenrich et al. (2011) improved upon Carollo et al.'s study. These new works, which are disputed by Beers et al. (2011), found no evidence for a dichotomous halo, particularly in regard to the kinematics and density profile. Our measurements for NGC 2419 additionally provide evidence against a dichotomy in detailed abundances. The outer halo GCs show the same bulk abundance properties-and, by extension, (short) star formation timescales-as the inner halo GCs.

We are grateful to the many people who have worked to make the Keck Telescope and its instruments a reality and to operate and maintain the Keck Observatory. The authors extend special thanks to those of Hawaiian ancestry on whose sacred mountain we are privileged to be guests. Without their generous hospitality, none of the observations presented herein would have been possible. We thank Alex Heger and Ken Nomoto for helpful conversations. J.G.C. and W.H. thank NSF grants AST0507219 and AST-0908139 for partial support. Work by E.N.K. was supported by NASA through Hubble Fellowship grand HST-HF-01233.01 awarded to E.N.K. by the Space Telescope Science institute, which is operated by the Association of Universities for Research in Astronomy, Inc., for NASA, under contract NAS 5-26555.

\section{REFERENCES}

Alcaino, G., \& Liller, W. 1980, AJ, 85, 1330

Andrievsky, S. M., Spite, M., Korotin, S. A., Spite, F., \& Bonifacio, P. 2008, A\&A, 481, 481

Andrievsky, S. M., Spite, M., Korotin, S. A., et al. 2010, A\&A, 509, 88

Baumgardt, H., Côté, P., Rejkuba, M., et al. 2009, MNRAS, 396, 2051

Baumuller, D. G., \& Gehren, T. 1996, A\&A, 307, 961

Beers, T. C., Carollo, D., Ivezic, Z., et al. 2011, ApJ, submitted (arXiv:1104.2513)

Bolte, M. 1987, ApJ, 319, 760

Briley, M. M., Cohen, J. G., \& Stetson, P. B. 2002, ApJ, 579, L17

Brown, J. A., Wallerstein, G., \& Zucker, D. 1997, AJ, 114, 180

Carollo, D., Beers, T. C., Lee, Y. S., et al. 2007, Nature, 450, 1020

Carretta, E., Bragaglia, A., Gratton, R., D’Orazi, V., \& Lucatello, S. 2009a, A\&A, 508, 695

Carretta, E., Bragaglia, A., Gratton, R., \& Lucatello, S. 2009b, A\&A, 505, 139

Carretta, E., Bragaglia, A., Gratton, R. G., et al. 2009c, A\&A, 505, 117

Carretta, E., Bragaglia, A., Gratton, R., et al. 2010, ApJ, 722, L1

Casagrande, L., Ramirez, I., Melendez, J., Bessell, M., \& Asplund, M. 2010, A\&A, 512, A54

Cohen, J. G. 2004, AJ, 127, 1545

Cohen, J. G., \& Huang, W. 2009, ApJ, 701, 1053

Cohen, J. G., \& Huang, W. 2010, ApJ, 719, 931

Cohen, J. G., Kirby, E. N., Simon, J., \& Geha, M. 2010, ApJ, 725, 288

Cohen, J. G., \& Melendez, J. 2005a, AJ, 129, 303

Cohen, J. G., \& Melendez, J. 2005b, AJ, 129, 1607

Conroy, C., Loeb, A., \& Spergel, D. 2010, ApJL, submitted (arXiv:1010.5783)

Cutri, R. M., et al. 2003, Explanatory Supplement to the 2MASS All-Sky Data Release, http://www.ipac.caltech.edu/2mass/releases/allsky/doc/explsup. html

D’Orazi, V., Gratton, R., Lucatello, S., et al. 2010, ApJ, 719, L213

de Jong, J. T. A., Yanny, B., Rix, H. W., et al. 2010, ApJ, 714, 663

Gratton, R., Sneden, C., \& Carretta, E. 2004, ARA\&A, 42, 385

Harris, W. E. 1996, AJ, 112, 1487

Harris, W. E., Bell, R. A., Vandenberg, D. A., et al. 1997, AJ, 114, 1030

Heger, A., \& Woosley, S. E. 2010, ApJ, 724, 341

Houdashelt, M. L., Bell, R. A., \& Sweigart, A. V. 2000, AJ, 119, 1448

Ibata, R. A., Gilmore, G. F., \& Irwin, M. J. 1995, MNRAS, 277, 781

Irwin, M. 1999, in ASP Conf. Ser. 192, The Stellar Content of Local Group Galaxies, ed. P. Whitelock \& R. Cannon (San Francisco, CA: ASP), 409

Ivans, I. I., Sneden, C., James, C. R., et al. 2003, ApJ, 592, 906

Kim, Y.-C., Demarque, P., Yi, S. K., \& Alexander, D. R. 2002, ApJS, 143, 499

Kirby, E. N., Cohen, J. G., Smith, G. H., et al. 2011, ApJ, 727, 79

Koch, A., \& Côté, P. 2010, A\&A, 517, A59

Koch, A., Côté, P., \& McWilliam, A. 2009, A\&A, 506, 729

Kurucz, R. L. 1993, ATLAS9 Stellar Atmosphere Programs and 2 km/s Grid (Kurucz CD-ROM No. 13)

Law, D. R., \& Majewski, S. R. 2010, ApJ, 718, 1128

Lawler, J. E., Wickliffe, M. E., Den Hartog, E. A., \& Sneden, C. 2001, ApJ, 563,1075

Mackey, A. D., \& van den Bergh, S. 2005, MNRAS, 360, 631

Masonkina, L., Gehren, T., \& Bikmaev, I. 2000, A\&A, 343, 519

Newberg, H. J., Yanny, B., Grebel, E. K., et al. 2003, ApJ, 596, L191

Nomoto, K., Tominaga, N., Umeda, H., Kobayashi, C., \& Maeda, K. 2006, Nucl. Phys. A, 777, 424

Prochaska, J. X., Naumov, S. O., Carney, B. W., McWilliam, A., \& Wolfe, A. M. 2000, AJ, 120, 2513

Ralchenko, Yu., Kramida, A. E., Reader, J., \& the NIST ASD Team 2010, NIST Atomic Spectra Database (version 4.0), http://physics.nist.gov/asd

Sarajedini, A., \& Layden, A. C. 1995, AJ, 109, 1086 
Sbordone, L., Bonifacio, P., Marconi, G., Buonanno, R., \& Zaggia, S. 2005, A\&A, 437, 905

Schoenrich, R., Asplund, M., \& Casagrande, L. 2011, MNRAS

Sesar, B., Juric, M., \& Ivezic, Z. 2011, ApJ, 731, 4

Shetrone, M., Côté, P., \& Sargent, W. L. W. 2001, ApJ, 568, 592

Shetrone, M., Venn, K. A., Tolstoy, E., et al. 2003, AJ, 125, 648

Skrutskie, M. F., Cutri, R. M., Stiening, R., et al. 2006, AJ, 131, 1163

Sneden, C. 1973, PhD thesis, Univ. Texas
Stetson, P. B. 2005, PASP, 117, 563

Suntzeff, N., Kraft, R. P., \& Kinman, T. D. 1988, AJ, 95, 91

Takeda, Y., Kaneko, H., Matsumoto, N., et al. 2010, PASJ, 61, 563

Takeda, Y., Zhao, G., Takada-Hidai, M., et al. 2003, Chin. J. Astron. Astrophys, 3,311

van den Bergh, S., \& Mackey, D. 2004, MNRAS, 354, 713

Vogt, S. E., Allen, S. L., Bigelow, B. C., et al. 1994, Proc. SPIE, 2198, 362

Woosley, S. E., \& Weaver, T. A. 1995, ApJS, 101, 181 